\title{
Synthesis of a Novel Series of 4-Arylpiperazinyl Derivatives Linked to a 2-(Pyridin-3-yl)-1H-benzimidazole as New Delavirdine Analogues
}

\author{
David Pessoa-Mahana, ${ }^{*, a}$ Andrés Núñez, ${ }^{a}$ Christian Espinosa, ${ }^{a}$ Jaime Mella-Raipán ${ }^{a}$ \\ and Hernán Pessoa-Mahana ${ }^{b}$ \\ ${ }^{a}$ Departamento de Farmacia, Facultad de Química, Pontificia Universidad Católica de Chile, \\ Casilla 306, Santiago 22, Chile \\ ${ }^{b}$ Departamento de Química Orgánica y Físico-Química, Facultad de Ciencias Químicas y \\ Farmacéuticas, Universidad de Chile, Casilla 233, Santiago 1, Chile
}

A síntese de uma série de arilpiperazinas ligadas à estrutura 2-(piridina-3-il)-1H-benzo $[d]$ imidazol, através de um linker alquílico é relatada. Os novos derivados 1-(2-(4-arilpiperazina-1il)alquil)-2-(piridina-3-il)-1H-benzimidazol são estruturalmente relacionados à droga anti-HIV-1 Delavirdina e pertencem à família bis(heteroaril)piperazinas (BHAPs), um conhecido grupo de inibidores da transcriptase reversa HIV-1.

The synthesis of a series of substituted arylpiperazines linked to a 2-(pyridin-3-yl)-1H-benzo $[d]$ imidazole scaffold through an alkylic linker is reported. The novel 1-(2-(4-arylpiperazin-1-yl) alkyl)-2-(pyridin-3-yl)-1H-benzimidazole derivatives are structurally related to the anti-HIV-1 drug Delavirdine and belong to the bis(heteroaryl)piperazines family (BHAPs), a well known HIV-1 reverse transcriptase inhibitors group.

Keywords: arylpiperazinyl derivatives, benzo $[d]$ imidazole, delavirdine analogues, alkylation

\section{Introduction}

Non nucleoside HIV-1 reverse transcriptase inhibitors (NNRTIs) such as nevirapine, have been approved for the treatment of acquired immune deficiency syndrome (AIDS). ${ }^{1}$ A major limitation of such treatment is the emergence of resistant virus with specific mutations in the reverse transcriptase (RT) gene. Therefore, the development of new active compounds has been the focus in many laboratories. ${ }^{2,3}$
The efforts along this line led to the discovery of the bis-(heteroaryl)piperazine (BHAP) class of HIV-1 reverse transcriptase inhibitors. ${ }^{4,5}$ Delavirdine, a BHAP derivative and first-generation NNRTI is displayed in Figure $1 .^{6}$

A variety of papers reporting the synthesis of Delavirdine analogues have been published up to the date. ${ }^{7,8}$ However, structural modifications have been mainly focused on the 4-heteroaryl piperazyne moiety, being the substitution of the indol ring by other heterocycles less explored. ${ }^{9}$ To

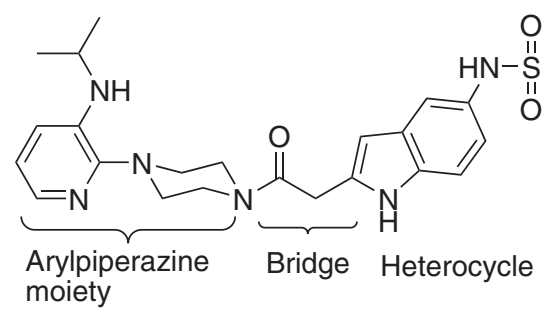

DELAVIRDINE

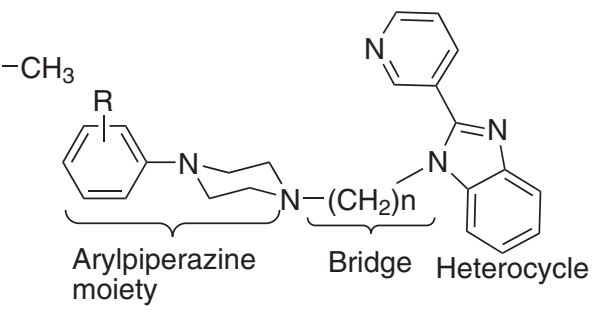

TARGET COMPOUNDS

Figure 1. Common structural pattern shared by Delavirdine and the target compounds. 
the best of our knowledge, there is not any report about the synthesis of BHAPs containing benzimidazole as the heterocyclic framework. The main reason we adopted to use benzimidazole framework, is the well known bioisosteric equivalence of this ring, respect to $1(\mathrm{H})$-Indole. Besides, preliminary docking studies carried out by our research group supported this bioisosteric change.

In this paper, we describe the synthesis of a new BHAP series combining two scaffolds, the 2-(pyridin-3-yl)-1Hbenzo $[d]$ imidazole and substituted 4-arylpiperazines linked by methilenic spacers. The structural similitude between Delavirdine and the target compounds is displayed in Figure 1.

\section{Results and Discussion}

The Delavirdine analogues $\mathbf{5}(\mathbf{a}-\mathbf{k})$ were prepared according to the retrosynthetic strategy displayed in scheme 1. We developed a general and useful synthesis of substituted 4-arylpiperazinyl ethyl (propyl) alcohol scaffolds 3(a-k), which were utilized for coupling reactions with 2-(pyridin-3-yl)-1H-benzo[ $d]$ imidazole 4 leading to the desires substituted target compounds.
The intermediate arylpiperazinyl building blocks $\mathbf{3}(\mathbf{a}-\mathbf{k})$ were synthesized as follows. Treatment of the appropriate substituted arylpiperazine $\mathbf{1}$ with 1-bromoethanol (1-bromopropanol) in acetone at room temperature, gave the corresponding alcohols 2 (a-k) in 40-54\% yield along with the ethers $\mathbf{6}$ and 7 as minority side products (Scheme 2). The alcohols were easily purified by column chromatography, showing the characteristics hydroxyl spectral signals (IR:3590-3644 cm-1; ${ }^{1} \mathrm{H}-\mathrm{NMR}$, broad singlet $\left.\delta_{\mathrm{OH}} 2.86-3.87\right)$. The ${ }^{1} \mathrm{H}-\mathrm{NMR}$ spectra displayed also the piperazine and the methylene protons at high field $\left(\delta_{\mathrm{CH} 2} 1.70-3.87\right)$, supporting the presence of these functions.

Reaction of the alcohols $\mathbf{2 a - k}$ with mesyl chloride and triethylamine in dichlorometane at $0{ }^{\circ} \mathrm{C}$ provided the respective mesylated alcohols $\mathbf{3 a - k}$ in moderate to good yields (Scheme 2). The mesylation was mainly deduced by their IR spectra (absence of the hydroxyl signal and presence of the two characteristic $\mathrm{SO}_{2}$ signals at 1348 and $1173 \mathrm{~cm}^{-1}$ ) and their ${ }^{1} \mathrm{H}-\mathrm{NMR}$ spectra which displayed the singlet for the methyl group protons at $\delta: 2.78-3.08 \mathrm{ppm}$. Interestingly, the chloroethyl arylpiperazines $\mathbf{3 b}$ ' and $\mathbf{3 e}$ ' were obtained instead of the expected mesylated products.

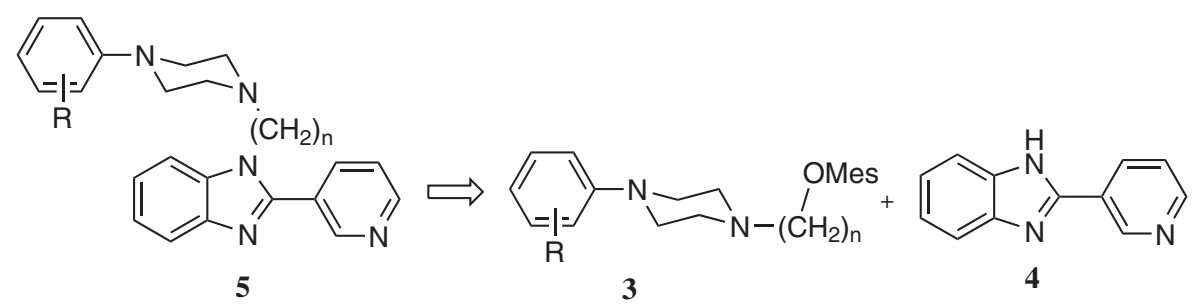

Scheme 1. Retrosynthetic strategy for the synthesis of $\mathbf{5}(\mathbf{a}-\mathbf{k})$.

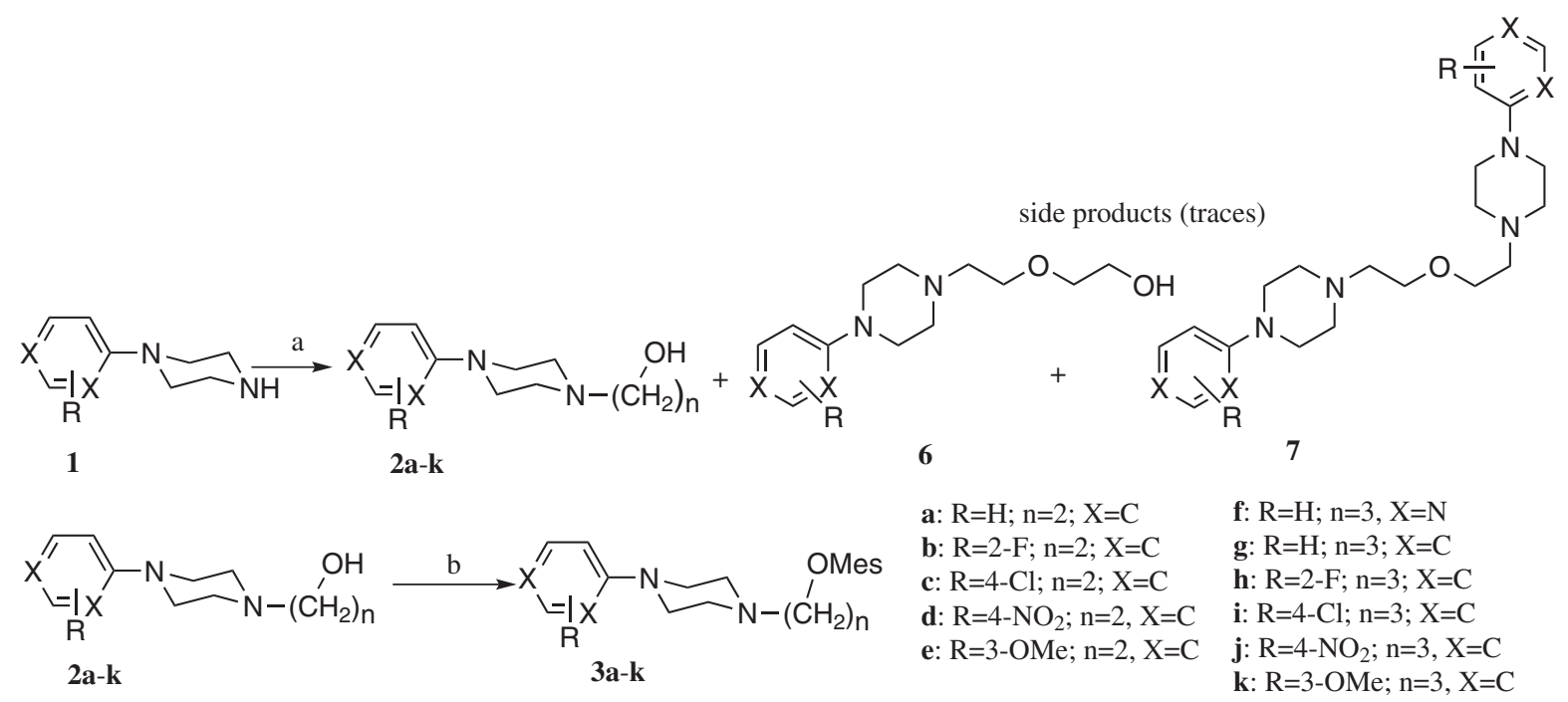

Scheme 2. Synthesis of Phenylpiperazinyl alkyl methanesulphonates intermediates and side products detected as traces. Reagents and conditions. a. 1,2-Bromoethanol or 1,3-bromopropanol, acetone, triethylamine, continuous stirring, RT, $24 \mathrm{~h}$; b. Methanesulfonyl chlorhide, dichloromethane, triethylamine, $0-5^{\circ} \mathrm{C}$, continuous stirring, RT, $4 \mathrm{~h}$. 


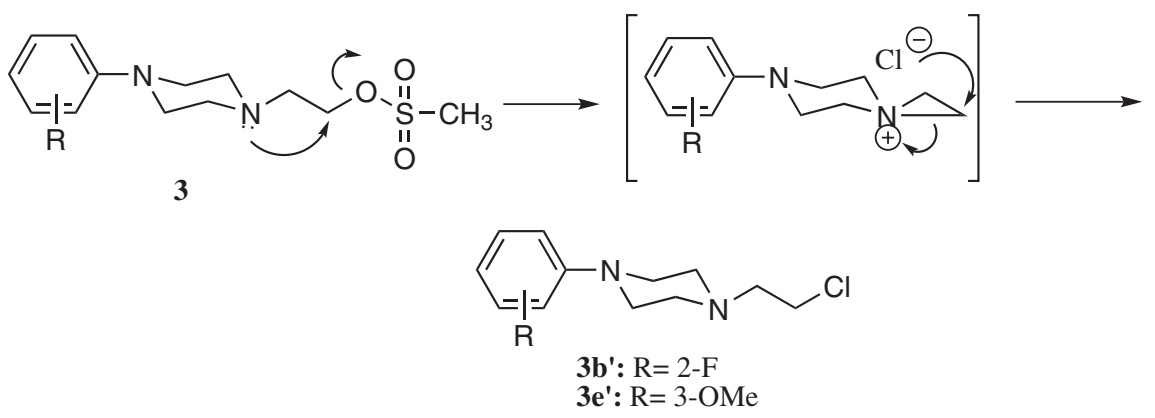

Scheme 3. A reasonable mechanistic proposal for the formation of chloroethyl arylpipera-zines $\mathbf{3 e}$ and $\mathbf{3 b}$.

A plausible mechanistic pathway (Scheme 3) probably involves the formation of an aziridinium intermediate generated by intramolecular attack of the basic piperazinic nitrogen followed by an $\mathrm{SN}_{2}$ attack of the chloride anion.

Benzimidazole 4 was efficiently obtained by condensation of $o$-phenylenediamine 8 with 3-pyridinecarboxaldehyde in ethanol at room temperature. According to the literature, ${ }^{8}$ the generation of benzimidazole $\mathbf{4}$ may be assumed to proceed through a three-step sequence: a) nucleophilic attack of the amino group onto the carbonylic carbon to give the imine intermediate $\mathbf{9}$, b) intramolecular cyclization of 9 to the benzimidazolydine intermediate 10, and c) aerobic oxidation to provide the benzimidazole ring 4 (Scheme 4).

Alkylation of the obtained benzimidazole moiety was readily accomplished using $\mathrm{NaOH}$ as the base in acetonitrile. As is shown is scheme 4, coupling of the benzimidazole anion with substituted alkyl arylpiperazines in the presence of triethylamine in acetonitrile at room temperature for $12 \mathrm{~h}$, provided target compounds $\mathbf{5}(\mathbf{a}-\mathbf{k})$ (Table 1) in low to moderate yields. ${ }^{1} \mathrm{H}-\mathrm{NMR}$ analysis of 5a displayed the four characteristic high field signals corresponding to the methylene protons of piperazine ring $(\delta: 3.10$ and $2.50 \mathrm{ppm})$ and the linker chain $(\delta: 4.40$ and
Table 1. Novel arylpiperazinil-benzimidazole derivatives obtained. Yields are expressed for pure products

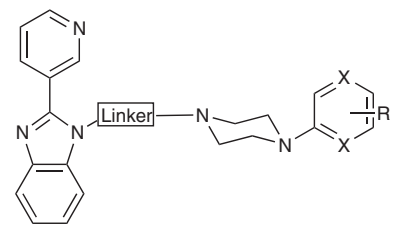

Chemical Data for Compounds 5a-k

\begin{tabular}{cccccc}
\hline Entry & Linker & $\mathrm{R}$ & $\mathrm{X}$ & Formula & Yield /\% \\
\hline $5 \mathrm{a}$ & $-\left(\mathrm{CH}_{2}\right)_{2}-$ & $\mathrm{H}$ & $\mathrm{C}$ & $\mathrm{C}_{24} \mathrm{H}_{25} \mathrm{~N}_{5}$ & 45 \\
$5 \mathrm{~b}$ & $-\left(\mathrm{CH}_{2}\right)^{-}$ & $2-\mathrm{F}$ & $\mathrm{C}$ & $\mathrm{C}_{24} \mathrm{H}_{24} \mathrm{FN}_{5}$ & 21 \\
$5 \mathrm{c}$ & $-\left(\mathrm{CH}_{2}\right)_{2}-$ & $4-\mathrm{Cl}$ & $\mathrm{C}$ & $\mathrm{C}_{24} \mathrm{H}_{22} \mathrm{ClN}_{5}$ & 35 \\
$5 \mathrm{~d}$ & $-\left(\mathrm{CH}_{2}\right)^{-}$ & $4-\mathrm{NO}_{2}$ & $\mathrm{C}$ & $\mathrm{C}_{24} \mathrm{H}_{24} \mathrm{~N}_{6} \mathrm{O}_{2}$ & 46 \\
$5 \mathrm{e}$ & $-\left(\mathrm{CH}_{2}\right)_{2}-$ & $3-\mathrm{OMe}$ & $\mathrm{C}$ & $\mathrm{C}_{25} \mathrm{H}_{27} \mathrm{~N}_{5} \mathrm{O}$ & 20 \\
$5 \mathrm{f}$ & $-\left(\mathrm{CH}_{2}\right)_{2}-$ & $\mathrm{H}$ & $\mathrm{N}$ & $\mathrm{C}_{22} \mathrm{H}_{23} \mathrm{~N}_{7}$ & 38 \\
$5 \mathrm{~g}$ & $-\left(\mathrm{CH}_{2}\right)_{3}-$ & $\mathrm{H}$ & $\mathrm{C}$ & $\mathrm{C}_{25} \mathrm{H}_{27} \mathrm{~N}_{5}$ & 48 \\
$5 \mathrm{~h}$ & $-\left(\mathrm{CH}_{2}\right)_{3}-$ & $2-\mathrm{F}$ & $\mathrm{C}$ & $\mathrm{C}_{25} \mathrm{H}_{26} \mathrm{FN}_{5}$ & 28 \\
$5 \mathrm{i}$ & $-\left(\mathrm{CH}_{2}\right)_{3}-$ & $4-\mathrm{Cl}$ & $\mathrm{C}$ & $\mathrm{C}_{25} \mathrm{H}_{26} \mathrm{ClN}_{5}$ & 38 \\
$5 \mathrm{j}$ & $-\left(\mathrm{CH}_{2}\right)_{3}-$ & $4-\mathrm{NO}$ & $\mathrm{C}$ & $\mathrm{C}_{25} \mathrm{H}_{26} \mathrm{~N}_{6} \mathrm{O}_{2}$ & 24 \\
$5 \mathrm{k}$ & $-\left(\mathrm{CH}_{2}\right)_{3}-$ & $3-\mathrm{OMe}$ & $\mathrm{C}$ & $\mathrm{C}_{26} \mathrm{H}_{29} \mathrm{~N}_{5} \mathrm{O}$ & 33 \\
\hline
\end{tabular}

$2.84 \mathrm{ppm}$ ), along with four aromatic doublets at low field $(\delta: 9.10,8.78,8.22$ and $7.92 \mathrm{ppm})$ typical of the pyridine ring attached to the $1 H$-benzimidazole system.
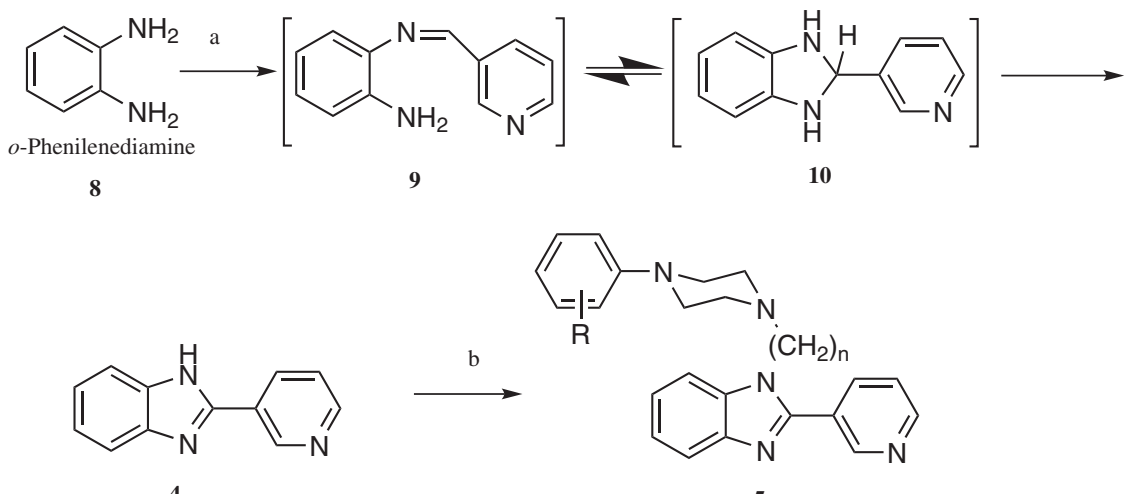

Scheme 4. Coupling of the benzimidazole moiety with substituted alkyl arylpiperazines. Reagents and conditions: a) 3-pyridincarboxaldehyde, ethanol, room temp., $48 \mathrm{~h}$; b) 2-(4-phenylpiperazin-1-yl)alkl methanesulfonates or 1-(2-chloroethyl)-4-phenylpiperazines, acetonitrile, continuous stirring, RT, $12 \mathrm{~h}$. 
In summary, a convenient method for the synthesis of 1-(2-(4-Arylpiperazin-1-yl)alkyl)-2-(pyridin-3yl)-1H-benzimidazole derivatives has been provided. Further studies on the application will be reported in due course.

\section{Experimental}

All organic solvents used for the synthesis were of analytical grade. IR spectra were recorded on a Brucker Vector 22 spectrophotometer using $\mathrm{KBr}$ discs. ${ }^{1} \mathrm{H}$ and ${ }^{13} \mathrm{C}$ NMR spectra were obtained on Brucker APC-200 spectrometer using tetramethylsilane as internal reference. Column chromatography was performed on Merck silica gel 60 (70-230 mesh). Thin layer chromatography separations were performed on Merck Kiesselgel 60 (70-230 mesh). Elemental analyses were carried out on a FISONS EA 1108 CHNS-O analyzer.

Synthesis of arylpiperazinyl alcohols 2(a-k). General procedure

A mixture of the commercial 4-arylpiperazines (6 mmol) and bromoethanol (bromopropanol) $(6 \mathrm{mmol})$ and triethylamine $(6 \mathrm{mmol})$ was stirred for $24 \mathrm{~h}$ in acetone $(30 \mathrm{~mL})$ at room temperature. The precipitate was filtered off and the filtrate was extracted with ethyl acetate $(3 \times 50 \mathrm{~mL})$, subsequently dried over anhydrous $\mathrm{MgSO}_{4}$ and evaporated under vacuum conditions. Column chromatography of the residue over silica gel (eluent EtOAc) afforded the corresponding alcohols. Spectral data for 2-(4-Arylpiperazin-1-yl) alcohols derivatives $\mathbf{2 a - 2 c}, \mathbf{2 e}, \mathbf{2 g}-\mathbf{2 i}$ and $\mathbf{2 k}$ were consistent with the assigned structures. ${ }^{10}$

\section{2-[4-(4-Nitrophenyl)piperazin-1-yl]ethanol (2d)}

Yellow oil (40\%), IR $v_{\max } / \mathrm{cm}^{-1}: 3590(\mathrm{OH}), 1529$ $\left(\mathrm{NO}_{2}\right), 1292\left(\mathrm{NO}_{2}\right) .{ }^{1} \mathrm{H}-\mathrm{NMR}\left(\mathrm{CDCl}_{3}\right) \delta 7.99(\mathrm{~d}, 2 \mathrm{H}, J$ $8.1 \mathrm{~Hz}, \mathrm{H}-3,5), 6.78$ (d, 2H, J 8.9Hz, H-2,6), $3.63(\mathrm{t}, 2 \mathrm{H}$, $J$ 9.8Hz, $\left.\mathrm{CH}_{2}-1\right), 3.57-2.89$ (m, 5H, $2 \times \mathrm{CH}_{2}$ pip., $\mathrm{OH}$ ), 2.59-2.55 (m, 6H, $2 \times \mathrm{CH}_{2}$ pip., $\left.\mathrm{CH}_{2}-2\right) .{ }^{13} \mathrm{C}-\mathrm{NMR}\left(\mathrm{CDCl}_{3}\right)$ $\delta 154.7,137.8,2 \times 25.8,2 \times 112.5,59.6,58.2,2 \times 52.5,2 \times$ 46.8. Anal. Calc. for $\mathrm{C}_{12} \mathrm{H}_{17} \mathrm{~N}_{3} \mathrm{O}_{3}: \mathrm{C}, 57.36 ; \mathrm{H}, 6.82 ; \mathrm{N}$, 16.72. Found: C, 57.70; H, 7.01; N, 16.66\%.

\section{2-(4-(Pyrazin-2-yl)piperazin-1-yl)ethanol (2f)}

Yellow oil (44\%), IR $v_{\text {max }} / \mathrm{cm}^{-1}: 3644(\mathrm{OH}), 1348(\mathrm{C}-\mathrm{N})$; ${ }^{1} \mathrm{H}-\mathrm{NMR}\left(\mathrm{CDCl}_{3}\right) \delta 8.39$ (s, 1H, H-2), 7.97 (m, 1H, H-6), 7.49 (d, $1 \mathrm{H}, J 6.24 \mathrm{~Hz}, \mathrm{H}-5), 3.56-3.51\left(\mathrm{~m}, 6 \mathrm{H}, 2 \times \mathrm{CH}_{2}\right.$ pip., $\left.\mathrm{CH}_{2}-1\right), 2.86$ (1H, b.s., $\mathrm{OH}$ ), $2.60-2.55$ (m, $6 \mathrm{H}, 2 \times \mathrm{CH}_{2}$ pip., $\left.\mathrm{CH}_{2}-2\right) ;{ }^{13} \mathrm{C}-\mathrm{NMR}\left(\mathrm{CDCl}_{3}\right) \delta 157.7,130.9,110,65.1$,
62.7, 57.8, 2×52.8, $2 \times 46.6$. Anal. Calc. for $\mathrm{C}_{10} \mathrm{H}_{16} \mathrm{~N}_{4} \mathrm{O}: \mathrm{C}$, 57.67; H, 7.74; N, 26.90. Found: C, 57.55; H, 7.75; N, $26.82 \%$.

\section{3-[4-(4-Nitrophenyl)piperazin-1-yl]propan-1-ol (2j)}

Yellow oil (54\%), IR $v_{\max } / \mathrm{cm}^{-1}: 3630(\mathrm{OH}), 1527\left(\mathrm{NO}_{2}\right)$; ${ }^{1} \mathrm{H}-\mathrm{NMR}\left(\mathrm{CDCl}_{3}\right) \delta 8.11(\mathrm{~d}, 2 \mathrm{H}, J 11 \mathrm{~Hz}, \mathrm{H}-3,5), 6.80$ (d, $2 \mathrm{H}, J 9.4 \mathrm{~Hz}, \mathrm{H}-2,6), 3.87-3.84\left(\mathrm{~m}, 3 \mathrm{H}, \mathrm{CH}_{2}-1, \mathrm{OH}\right)$, 3.53-3.44 (m, 4H, $2 \times \mathrm{CH}_{2}$-pip.), 2.60 (m, $6 \mathrm{H}, 2 \times \mathrm{CH}_{2}$-pip., $\left.\mathrm{CH}_{2}-3\right), 1.70\left(\mathrm{~m}, 2 \mathrm{H}, \mathrm{CH}_{2}-2\right) ;{ }^{13} \mathrm{C}-\mathrm{NMR}\left(\mathrm{CDCl}_{3}\right) \delta 154.7$, 138.5, $2 \times 125.9,2 \times 112.7,58.3,2 \times 52.7,52.4,2 \times 46.9,28.7$. Anal. Calc. for $\mathrm{C}_{13} \mathrm{H}_{19} \mathrm{~N}_{3} \mathrm{O}_{3}:$ C, 58.85; H, 7.22; N, 15.84; Found: C, 58.52; H, 7.34; N, 16.01\%.

Synthesis of 2-(4-Arylpiperazinyl) alkyl methanesulphonate derivatives 3(a-k). General procedure

Alcohols 2a-k (2.4 mmol) were added to a solution of methanesulfonyl chloride $(2.5 \mathrm{mmol})$ in dichloromethane $(15 \mathrm{~mL})$ and triethylamine $(2.5 \mathrm{mmol})$ at $0{ }^{\circ} \mathrm{C}$. The mixture was maintained with stirring for $4 \mathrm{~h}$ and then concentrated in vacuo. Purification of the crude by column chromatography $\mathrm{CH}_{2} \mathrm{Cl}_{2}$ / AcOEt (1:2) as the eluent, afforded the title compounds (3a-k) as yellow oils in moderated yields (35-50\%).

2-(4-Phenylpiperazin-1-yl)ethyl methanesulphonate (3a) (48\%), IR $v_{\max } / \mathrm{cm}^{-1}: 1347\left(\mathrm{SO}_{2}\right), 1172\left(\mathrm{SO}_{2}\right) ;{ }^{1} \mathrm{H}-\mathrm{NMR}$ $\left(\mathrm{CDCl}_{3}\right) \delta 7.30(\mathrm{t}, 2 \mathrm{H}, J 8.0 \mathrm{~Hz}, \mathrm{H}-3,5), 6.94(\mathrm{~m}, 3 \mathrm{H}$, $\mathrm{H}-2,4,6), 3.83$ (t, $\left.2 \mathrm{H}, J 7.0 \mathrm{~Hz}, \mathrm{CH}_{2}-1\right), 3.44-3.20$ (m, 4H, $2 \times \mathrm{CH}_{2}$ pip.), 2.98 (s, $\left.3 \mathrm{H}, \mathrm{CH}_{3}\right), 2.89$ (t, $2 \mathrm{H}, J 7.2 \mathrm{~Hz}, \mathrm{CH}_{2}-$ 2), $2.70\left(\mathrm{t}, 4 \mathrm{H}, J 5.1 \mathrm{~Hz}, 2 \times \mathrm{CH}_{2}\right.$ pip.); ${ }^{13} \mathrm{C}-\mathrm{NMR}\left(\mathrm{CDCl}_{3}\right) \delta$ 151.2, $2 \times 129.3,119.9,2 \times 116.1,65.3,59.8,2 \times 53.2,2 \times 49.4$, 37.1. Anal. Calc. for $\mathrm{C}_{13} \mathrm{H}_{20} \mathrm{~N}_{2} \mathrm{O}_{3} \mathrm{~S}: \mathrm{C}, 54.91 ; \mathrm{H}, 7.09 ; \mathrm{N}$, 9.85. Found: C, 55.02; H, 7.11; N, 10.02\%.

\section{1-(2-Chloroethyl)-4-(2-fluorophenyl)piperazine (3b')}

(35\%), IR $v_{\max } / \mathrm{cm}^{-1}: 1007$ (C-F), $728(\mathrm{C}-\mathrm{Cl}) ;{ }^{1} \mathrm{H}-\mathrm{NMR}$ $\left(\mathrm{CDCl}_{3}\right) \delta$ 7.18-6.85 (m, 3H, H-3,4,5), 6.68-6.57 (m, 1H, $\mathrm{H}-6$ ), 3.73 (t, $\left.2 \mathrm{H}, J 13.5 \mathrm{~Hz}, \mathrm{CH}_{2}-1\right), 3.25$ (t, $4 \mathrm{H}, J 9.5 \mathrm{~Hz}$, $2 \times \mathrm{CH}_{2}$ pip.), 2.89 (m, $6 \mathrm{H}, 2 \times \mathrm{CH}_{2}$ pip., $\left.\mathrm{CH}_{2}-2\right) ;{ }^{13} \mathrm{C}-\mathrm{NMR}$ $\left(\mathrm{CDCl}_{3}\right) \delta 158.16\left(\mathrm{~d}, 2 \mathrm{C}, J_{\mathrm{C}-\mathrm{F}} 337 \mathrm{~Hz}\right), 151,78,124.6$, 123.1, 122.9, 119.1, 116.4, 115.9, 59.5, 2×53.2, 2×49.7, 39.9. Anal. Calc. for $\mathrm{C}_{12} \mathrm{H}_{16} \mathrm{ClFN}_{2}: \mathrm{C}, 59.38 ; \mathrm{H}, 6.64 ; \mathrm{N}$, 11.54. Found: C, 59.49; H, 6.67; N, $11.38 \%$.

\section{2-[4-(4-Chlorophenyl)piperazin-1-yl]ethyl methane-} sulphonate $(3 \mathrm{c})$

(38\%), IR $v_{\max } / \mathrm{cm}^{-1}: 1349\left(\mathrm{SO}_{2}\right), 1173\left(\mathrm{SO}_{2}\right), 933(\mathrm{C}-$ $\mathrm{Cl}) ;{ }^{1} \mathrm{H}-\mathrm{NMR}\left(\mathrm{CDCl}_{3}\right) \delta 7.30(\mathrm{~d}, 2 \mathrm{H}, J 8.4 \mathrm{~Hz}, \mathrm{H}-3,5)$, 6.91 (d, 2H, J $8.4 \mathrm{~Hz}, \mathrm{H}-2,6), 4.18\left(\mathrm{~m}, 2 \mathrm{H}, \mathrm{CH}_{2}-1\right)$, 
3.52-3.37 (m, 4H, $2 \times \mathrm{CH}_{2}$ pip.), 3.32-2.80 (m, 9H, $2 \times \mathrm{CH}_{2}$ pip., $\left.\mathrm{CH}_{2}-2, \mathrm{CH}_{3}\right) ;{ }^{13} \mathrm{C}-\mathrm{NMR}\left(\mathrm{CDCl}_{3}\right) \delta 149.3,130.9$, $2 \times 129.4,2 \times 118.6,66.2,2 \times 52.6,2 \times 49.7,38.7,29.7$. Anal. Calc. for $\mathrm{C}_{13} \mathrm{H}_{19} \mathrm{ClN}_{2} \mathrm{O}_{3} \mathrm{~S}: \mathrm{C}, 48.97 ; \mathrm{H}, 6.01 ; \mathrm{N}, 8.79$. Found: C, 49.05; H, 6.14; N, 8.72\%.

\section{2-[4-(4-Nitrophenyl)piperazin-1-yl]ethyl methane- sulphonate $(\mathbf{3 d})$}

(35\%), IR $v_{\max } / \mathrm{cm}^{-1}: 1348\left(\mathrm{SO}_{2}\right), 1173\left(\mathrm{SO}_{2}\right), 1529,1292$ $\left(\mathrm{NO}_{2}\right)$.; ${ }^{1} \mathrm{H}-\mathrm{NMR}\left(\mathrm{CDCl}_{3}\right) \delta 8.20(\mathrm{~d}, 2 \mathrm{H}, J 7.9 \mathrm{~Hz}, \mathrm{H}-3,5)$, 6.90 (d, 2H, J 7.2 Hz, H-2,6), 3.81 (t, $2 \mathrm{H}, J 11.5 \mathrm{~Hz}, \mathrm{CH}_{2}-1$ ), 3.52-3.39 (m, $4 \mathrm{H}, 2 \times \mathrm{CH}_{2}$ pip), 3.29-2.78 (m, $9 \mathrm{H}, 2 \mathrm{xCH}_{2}$ pip., $\left.\mathrm{CH}_{2}-2, \mathrm{CH}_{3}\right) ;{ }^{13} \mathrm{C}-\mathrm{NMR}\left(\mathrm{CDCl}_{3}\right) \delta 153.8,137.5$, $2 \times 125.8,2 \times 113.7,63.4,55.2,2 \times 52.6,2 \times 50.1,37.9$. Anal. Calc. for $\mathrm{C}_{13} \mathrm{H}_{19} \mathrm{~N}_{3} \mathrm{O}_{5} \mathrm{~S}: \mathrm{C}, 47.41 ; \mathrm{H}, 5.81 ; \mathrm{N}, 12.76$. Found: C, 47.12; H, 5.61; N, $12.59 \%$.

\section{1-(2-Chloroethyl)-4-(3-methoxyphenyl)piperazine (3e')}

(37\%), IR $v_{\max } / \mathrm{cm}^{-1}: 2835\left(\mathrm{CO}-\mathrm{CH}_{3}\right), 728(\mathrm{C}-\mathrm{Cl})$; ${ }^{1} \mathrm{H}-\mathrm{NMR}\left(\mathrm{CDCl}_{3}\right) \delta 7.22\left(\mathrm{dd}, 1 \mathrm{H}, J_{1} 8.3 \mathrm{~Hz}, J_{2} 2.6 \mathrm{~Hz}\right.$, $\mathrm{H}-5)$, 6.61-6.22 (m, 3H, H-4,5,6), 3.82-3.71 (m, 5H, $\left.\mathrm{CH}_{2}-2, \mathrm{OCH}_{3}\right), 3.60$ (t, $\left.2 \mathrm{H}, J 11.9 \mathrm{~Hz}, \mathrm{CH}_{2}-1\right), 3.38-3.20$ (m, 4H, $2 \times \mathrm{CH}_{2}$-pip.), 2.67 (t, $4 \mathrm{H}, J 10 \mathrm{~Hz}, 2 \times \mathrm{CH}_{2}$-pip.); ${ }^{13} \mathrm{C}-\mathrm{NMR}\left(\mathrm{CDCl}_{3}\right) \delta 160.6,152.5,129.8,108.9,104.6$, 102.6, 97.34, 59.7, 55.2, $2 \times 53.1,2 \times 49.3,37.9$. Anal. Calc. for $\mathrm{C}_{14} \mathrm{H}_{19} \mathrm{ClN}_{2} \mathrm{O}: \mathrm{C}, 61.29 ; \mathrm{H}, 7.52 ; \mathrm{N}, 11.00$. Found: $\mathrm{C}$, $60.94 ; \mathrm{H}, 7.65 ; \mathrm{N}, 11.32 \%$.

2-(4-Pyrazin-2-yl-piperazin-1-yl)ethyl methanesulphonate (3f)

(37\%), IR $v_{\max } / \mathrm{cm}^{-1}: 1347\left(\mathrm{SO}_{2}\right), 1171\left(\mathrm{SO}_{2}\right) ;{ }^{1} \mathrm{H}-\mathrm{NMR}$ $\left(\mathrm{CDCl}_{3}\right) \delta{ }^{13} \mathrm{C}$ NMR $\left(\mathrm{CDCl}_{3}\right) \delta 8.35(\mathrm{~s}, 1 \mathrm{H}, \mathrm{H}-6), 8.08(\mathrm{~d}$, $1 \mathrm{H}, J 8 \mathrm{~Hz}, \mathrm{H}-4), 6.44$ (d, 1H, J $8.5 \mathrm{~Hz}, \mathrm{H}-3), 4.26$ (t, $2 \mathrm{H}$, $\left.J 11.0 \mathrm{~Hz}, \mathrm{CH}_{2}-1\right), 3.80$ (m, $4 \mathrm{H}, 2 \times \mathrm{CH}_{2}$ pip), 3.03-2.86 (m, 5H, $\mathrm{CH}_{2}-2, \mathrm{CH}_{3}$ ), 2.63-2.48 (m, 4H, $2 \times 2 \times \mathrm{CH}_{2}$ pip). ${ }^{13} \mathrm{C} \mathrm{RMN}\left(\mathrm{CDCL}_{3}\right) \delta 162.3,159.1,156.5,103.6,53.6$, $2 \times 52.0,2 \times 49.8,46.7,42.1$. Anal. Calc. for $\mathrm{C}_{11} \mathrm{H}_{18} \mathrm{~N}_{4} \mathrm{O}_{3} \mathrm{~S}$ : C, 46.14; H, 6.34; N, 19.57. Found: C, 45.94; H, 6.45; $\mathrm{N}, 19.82 \%$.

3-[4-(3-Methoxyphenyl)piperazin-1-yl]propyl methanesulphonate $(\mathbf{3 g})$

(35\%), IR $v_{\max } / \mathrm{cm}^{-1}: 1347\left(\mathrm{SO}_{2}\right), 1173\left(\mathrm{SO}_{2}\right) ;{ }^{1} \mathrm{H}-\mathrm{NMR}$ $\left(\mathrm{CDCl}_{3}\right) \delta 7.30(\mathrm{t}, 2 \mathrm{H}, J 8.4 \mathrm{~Hz}, \mathrm{H}-3,5), 6.96(\mathrm{~m}, 3 \mathrm{H}$, $\mathrm{H}-2,4,6), 4.31\left(\mathrm{~m}, 2 \mathrm{H}, \mathrm{CH}_{2}-1\right), 3.2\left(\mathrm{t}, 4 \mathrm{H}, J 5 \mathrm{~Hz}, 2 \times \mathrm{CH}_{2}-\right.$ pip.), 2.90 (s, $3 \mathrm{H}, \mathrm{CH}_{3}$ ), 2.63-2.43 (m, $6 \mathrm{H}, 2 \times \mathrm{CH}_{2}$-pip., $\left.\mathrm{CH}_{2}-3\right)$, 2.10-1.90 (m, $\left.2 \mathrm{H}, \mathrm{CH}_{2}-2\right) ;{ }^{13} \mathrm{C}-\mathrm{NMR}\left(\mathrm{CDCl}_{3}\right) \delta$ 160.6, 151.1, 129.5, 119.7, 116, 68.5, 55.0, 53.9, 2×53.1, $2 \times 48.9,37.8,26.9$. Anal. Calc. for $\mathrm{C}_{14} \mathrm{H}_{22} \mathrm{~N}_{2} \mathrm{O}_{3} \mathrm{~S}$ : C, 56.35; H, 7.43; N, 9.39; O. Found: C, 56.47; H, 7.76; $\mathrm{N}, 9.12 \%$.
3-[4-(2-Fluorophenyl)piperazin-1-yl]propyl methanesulphonate (3h)

(50\%), IR $v_{\max } / \mathrm{cm}^{-1}: 1348\left(\mathrm{SO}_{2}\right), 1170\left(\mathrm{SO}_{2}\right), 1010$ (C-F); ${ }^{1} \mathrm{H}-\mathrm{NMR}\left(\mathrm{CDCl}_{3}\right) \delta$ 7.28-6.87 (m, 4H, H-3,4,5,6), 3.82 (t, $\left.2 \mathrm{H}, J 10.2 \mathrm{~Hz}, \mathrm{CH}_{2}-1\right), 3.47$ (m, 4H, $2 \times \mathrm{CH}_{2}$-pip.), 3.36-2.92 (m, 9H, $2 \times \mathrm{CH}_{2}$-pip., $\mathrm{CH}_{2}-3, \mathrm{CH}_{3}$ ), 2.12-1.90 $\left(\mathrm{m}, 2 \mathrm{H}, \mathrm{CH}_{2}-2\right) ;{ }^{13} \mathrm{C}-\mathrm{NMR}\left(\mathrm{CDCl}_{3}\right) \delta 158.10\left(\mathrm{~d}, 2 \mathrm{C}, J_{\mathrm{C}-\mathrm{F}}\right.$ $318 \mathrm{~Hz}), 153.10,137.5,124.4,118.9,116.2,115.9,68.4$, 62.4, 59.4, 53.9, $2 \times 53.2,2 \times 45.5,39.7,26.7$. Anal. Calc. for $\mathrm{C}_{14} \mathrm{H}_{21} \mathrm{FN}_{2} \mathrm{O}_{3} \mathrm{~S}: \mathrm{C}, 53.15 ; \mathrm{H}, 6.69 ; \mathrm{N}, 8.85$. Found: $\mathrm{C}$, $52.73 ; \mathrm{H}, 6.66 ; \mathrm{N}, 8.24 \%$.

3-[4-(4-Chlorophenyl)piperazin-1-yl]propyl methanesulphonate (3i)

(50\%), IR V $v_{\text {max }} / \mathrm{cm}^{-1}: 1350\left(\mathrm{SO}_{2}\right), 1178\left(\mathrm{SO}_{2}\right), 938(\mathrm{C}-\mathrm{Cl})$; ${ }^{1} \mathrm{H}-\mathrm{NMR}\left(\mathrm{CDCl}_{3}\right) \delta 7.41$ (d, 2H, J 12.3 Hz, H-3,5), 6.94 (d, $2 \mathrm{H}, J 8.8 \mathrm{~Hz}, \mathrm{H}-2,6), 3.71$ (t, $\left.2 \mathrm{H}, J 5.8 \mathrm{~Hz}, \mathrm{CH}_{2}-1\right), 3.50$ (m, $4 \mathrm{H}, 2 \times \mathrm{CH}_{2}$-pip), 3.36-2.92 (m, $6 \mathrm{H}, 2 \times \mathrm{CH}_{2}$-pip., $\mathrm{CH}_{2}-3$ ), 2.87 $\left(\mathrm{s}, 3 \mathrm{H}, \mathrm{CH}_{3}\right), 2.25-2.00\left(\mathrm{~m}, 2 \mathrm{H}, \mathrm{CH}_{2}-2\right) ;{ }^{13} \mathrm{C}-\mathrm{NMR}\left(\mathrm{CDCl}_{3}\right) \delta$ $147.9,2 \times 129.4,126.9,2 \times 118.5,66.9,2 \times 52.1,2 \times 46.8,41.6$, 39.6, 26.5. Anal. Calc. for $\mathrm{C}_{14} \mathrm{H}_{21} \mathrm{ClN}_{2} \mathrm{O}_{3} \mathrm{~S}: \mathrm{C}, 50.52 ; \mathrm{H}, 6.36$; $\mathrm{N}$, 8.42. Found $\mathrm{C}_{14} \mathrm{H}_{21} \mathrm{ClN}_{2} \mathrm{O}_{3} \mathrm{~S}: \mathrm{C}, 49.97 ; \mathrm{H}, 6.72 ; \mathrm{N}, 8.14 \%$.

3-[4-(4-Nitrophenyl)piperazin-1-yl]propyl methanesulphonate (3j)

(46\%), IR v $v_{\max } / \mathrm{cm}^{-1}: 1351\left(\mathrm{SO}_{2}\right), 1173\left(\mathrm{SO}_{2}\right) ;{ }^{1} \mathrm{H}-\mathrm{NMR}$ $\left(\mathrm{CDCl}_{3}\right) \delta 8.11(\mathrm{~d}, 2 \mathrm{H}, J 6.5 \mathrm{~Hz}, \mathrm{H}-3,5), 6.80(\mathrm{~d}, 2 \mathrm{H}, J 9.5$ $\mathrm{Hz}, \mathrm{H}-2,6), 4.28$ (t, 2H, J 6.2 Hz, H-1), 3.65-3.43 (m, 4H, $2 \times \mathrm{CH}_{2}$-pip), 3.32-2.92 (m, 6H, $2 \times \mathrm{CH}_{2}$-pip., $\left.\mathrm{CH}_{2}-2\right), 2.88$ (s, $\left.3 \mathrm{H}, \mathrm{CH}_{3}\right), 2.26$ (t, $\left.2 \mathrm{H}, J 6.6 \mathrm{~Hz}, \mathrm{H}-2\right) ;{ }^{13} \mathrm{C}-\mathrm{NMR}\left(\mathrm{CDCl}_{3}\right)$ $\delta 150.8,137.9,2 \times 125.9,2 \times 112.7,68.12,2 \times 52.6,2 \times 46.9$, 37.5, 29.7. Anal. Calc. for $\mathrm{C}_{14} \mathrm{H}_{21} \mathrm{~N}_{3} \mathrm{O}_{5} \mathrm{~S}: \mathrm{C}, 48.97 ; \mathrm{H}, 6.16$; N, 12.24. Found: C, 49.14; H, 6.28; N, $12.09 \%$.

3-[4-(3-Methoxyphenyl)piperazin-1-yl]propyl methanesulphonate $(3 \mathrm{k})$

(37\%), IR v $v_{\max } / \mathrm{cm}^{-1}: 1349\left(\mathrm{SO}_{2}\right), 1170\left(\mathrm{SO}_{2}\right), 2835(\mathrm{C}-$ $\mathrm{H}) ;{ }^{1} \mathrm{H}-\mathrm{NMR}\left(\mathrm{CDCl}_{3}\right) \delta 7.22\left(\mathrm{dd}, 1 \mathrm{H}, J_{1} 8.2 \mathrm{~Hz}, J_{2} 2.86\right.$ Hz, H-5), 6.61-6.21 (m, 3H, H-4,5,6), 4.30 (t, 2H, J 6.3 Hz, $\mathrm{H}-1), 3.82$ (d, $3 \mathrm{H}, J 9.6 \mathrm{~Hz}, \mathrm{H}-2), 3.75$ (s, $\left.3 \mathrm{H}, \mathrm{OCH}_{3}\right), 3.25$ (t, $4 \mathrm{H}, J 9.8 \mathrm{~Hz}, 2 \times \mathrm{CH}_{2}$-pip.), 2.9-2.5 (m, $6 \mathrm{H}, \mathrm{CH}_{2}$-pip, $\left.\mathrm{CH}_{2}-3\right), 2.18(\mathrm{t}, 2 \mathrm{H}, J 6.7 \mathrm{~Hz}, \mathrm{H}-2) ;{ }^{13} \mathrm{C}-\mathrm{NMR}\left(\mathrm{CDCl}_{3}\right) \delta$ 160.4, 148.2, 129.8, 108.9, 104.6, 102.6, 68.3, 55.2, 53.9, $2 \times 53.1,2 \times 48.9,37.3,26.4$. Anal. Calc. for $\mathrm{C}_{15} \mathrm{H}_{24} \mathrm{~N}_{2} \mathrm{O} \mathrm{O}_{4} \mathrm{~S}: \mathrm{C}$, $54.86 ; \mathrm{H}, 7.37 ; \mathrm{N}, 8.53$. Found: C, 54.49; H, 7.65; N, 8.24\%.

Synthesis of 1-[2-(4-Arylpiperazin-1-il)alkyl]-2-(pyridin-3yl)-1H-benzimidazoles $\mathbf{5 a}-\boldsymbol{k}$. General procedures

A solution of 2-(pyridin-3-yl)- $1 H$-benzimidazole 4 $(0.15 \mathrm{~g}, 0.75 \mathrm{mmol})$ in acetonitrile was stirred with $\mathrm{NaOH}$ 
for 2 hours. The benzimidazole anion afforded was added to a solution of the corresponding arylpiperazinyl alkyl methanesulphonate derivatives $\mathbf{3}(0.70 \mathrm{mmol})$ in acetonitrile $(12 \mathrm{~mL})$. The mixture was stirred at room temperature for 12 hours and concentrated in vacuo. Purification of the crude by column chromatography with dichloromethane as the eluent afforded the target compounds $\mathbf{5 a - k}$ as yellow oils.

1-[2-(4-Phenylpiperazin-1-yl)ethyl]-2-pyridin-3-yl-1Hbenzimidazole $(5 a)$

(45\%), IR $v_{\max } / \mathrm{cm}^{-1}: 2885(\mathrm{C}-\mathrm{H}), 1338(\mathrm{C}-\mathrm{N}) .{ }^{1} \mathrm{H}-\mathrm{NMR}$ $\left(\mathrm{CDCl}_{3}\right) \delta 9.10(\mathrm{~d}, 1 \mathrm{H}, J 2.1 \mathrm{~Hz}, \mathrm{H}-2$ pyr.), 8.78 (d, 1H, J $4.9 \mathrm{~Hz}, \mathrm{H}-4$ pyr.), 8.22 (d, 1H, J 7.9 Hz, H-6 pyr.), 7.92 (d, 1H, J 8.5 Hz, H-5-pyr.), 7.53-7.14 (m, 6H, Ar), 6.85 (m, $3 \mathrm{H}, \mathrm{H}-2,4,6), 4.40\left(\mathrm{t}, 2 \mathrm{H}, J 6.5 \mathrm{~Hz}, \mathrm{CH}_{2}-1\right), 3.10(\mathrm{t}, 4 \mathrm{H}, J$ $4.9 \mathrm{~Hz}, 2 \times \mathrm{CH}_{2}$-pip.), 2.84 (t, $\left.2 \mathrm{H}, J 6.5 \mathrm{~Hz}, \mathrm{CH}_{2}-2\right), 2.50$ (t, $4 \mathrm{H}, J 5.1 \mathrm{~Hz}, 2 \times \mathrm{CH}_{2}$ pip.). ${ }^{13} \mathrm{C}-\mathrm{NMR}\left(\mathrm{CDCL}_{3}\right) \delta 151.3$, $150.7,149.9,143.3,137.2,135.6,2 \times 129.1,2 \times 123.6,123.4$, $2 \times 120.3,119.9,2 \times 116.1,110.2,57.2,2 \times 53.6,2 \times 48.9$, 42.9. Anal. Calc. for $\mathrm{C}_{24} \mathrm{H}_{25} \mathrm{~N}_{5}: \mathrm{C}, 75.17 ; \mathrm{H}, 6.57 ; \mathrm{N}, 18.26$. Found: C, 75.36; H, 6.42; N, 18.22\%.

1-\{2-[4-(2-Fluorophenyl)piperazin-1-yl]ethyl\}-2-pyridin3-yl-1H-benzimidazole (5b)

(21\%), IR $v_{\max } / \mathrm{cm}^{-1}: 1321(\mathrm{C}-\mathrm{N}), 1007(\mathrm{C}-\mathrm{F}) ;{ }^{1} \mathrm{H}-\mathrm{NMR}$ $\left(\mathrm{CDCl}_{3}\right) \delta 9.10$ (d, 1H, J 1.4Hz, H-2 pyr.), 8.78 (d, 1H, J $4.8 \mathrm{~Hz}, \mathrm{H}-4$ pyr.), 8.24 (d, 1H, J 7.9 Hz, H-6 pyr.), 7.92 (d, $1 \mathrm{H}, J$ 7.8 Hz, H-5-pyr.), 7.62-7.21 (m, 6H, Ar), 7.12-6.85 (m, 2H, H-4,6), 4.40 (t, 2H, J $\left.6.5 \mathrm{~Hz}, \mathrm{CH}_{2}-1\right), 2.97$ (t, 4H, $J 4.9 \mathrm{~Hz}, 2 \times \mathrm{CH}_{2}$-pip.), 2.84 (t, $\left.2 \mathrm{H}, J 6.5 \mathrm{~Hz}, \mathrm{CH}_{2}-2\right), 2.50$ (m, $4 \mathrm{H}, J 4.9 \mathrm{~Hz}, 2 \times \mathrm{CH}_{2}$ pip.). ${ }^{13} \mathrm{C}-\mathrm{NMR}\left(\mathrm{CDCl}_{3}\right) \delta 158.10$ $\left(\mathrm{d}, 2 \mathrm{C}, J_{\mathrm{C}-\mathrm{F}} 318 \mathrm{~Hz}\right), 153.10,151.1,150.7,149.9,143.2$, 137.2 , 135.6, 124.5, 124.4, 123.6, 123.4, 122.9, 122.7, $122.5,120.2,118.9,116.3,115.9,110.2,57.2,2 \times 53.7$, $2 \times 50.3$. Anal. Calc. for $\mathrm{C}_{24} \mathrm{H}_{24} \mathrm{FN}_{5}: \mathrm{C}, 71.80 ; \mathrm{H}, 6.03 ; \mathrm{N}$, 17.44. Found: C, 72.14; H, 6.14; N, $17.62 \%$.

1-\{2-[4-(4-Chlorophenyl)piperazin-1-yl]ethyl\}-2-pyridin3-yl-1H-benzimidazole (5c)

(35\%), IR $v_{\max } / \mathrm{cm}^{-1}: 1317(\mathrm{C}-\mathrm{N}), 933(\mathrm{C}-\mathrm{Cl}) ;{ }^{1} \mathrm{H}-\mathrm{NMR}$ $\left(\mathrm{CDCl}_{3}\right) \delta: 9.11(\mathrm{~d}, 1 \mathrm{H}, J 2.1 \mathrm{~Hz}, \mathrm{H}-2$ pyr. $), 8.78$ (d, 1H, $J$ $4.9 \mathrm{~Hz}, \mathrm{H}-4-$ pyr.), 8.25 (d, 1H, J 7.9 Hz, H-6 pyr.), 7.91 (d, 1H J 7.1 Hz, H-5-pyr.), 7.54-7.16 (m, 6H Ar) 7.11-6.87 (m, $2 \mathrm{H}, \mathrm{H}-2,6) 4.41$ (t, 2H, J $\left.6.4 \mathrm{~Hz}, \mathrm{CH}_{2}-1\right), 3.10$ (t, 4H, J 5 $\mathrm{Hz}, 2 \times \mathrm{CH}_{2}$-pip.), 2.88 (t, $\left.2 \mathrm{H}, J 12 \mathrm{~Hz}, \mathrm{CH}_{2}-2\right), 2.56$ (t, 4H, $J 9.8 \mathrm{~Hz}, 2 \times \mathrm{CH}_{2}$-pip.). ${ }^{13} \mathrm{C}$-NMR $\left(\mathrm{CDCl}_{3}\right) \delta 151.7,150.8$, 147.2, 137.2, 134.4, 132.5, 2×128.9, 124,4 123.6, 123.5, $123.4,122.9,120.3,2 \times 117.3,110.2,58.2,57.1,2 \times 53.5$, $2 \times 48.9,42.9$. Anal. Calc. for $\mathrm{C}_{24} \mathrm{H}_{22} \mathrm{ClN}_{5}$ : C, 68.97; H, 5.79; N, 16.76. Found: C, 69.02; H, 5.62; N, $17.00 \%$.
1-\{2-[4-(4-Nitrophenyl)piperazin-1-yl]ethyl\}-2-pyridin-3yl-1H-benzimidazole $(5 \mathbf{d})$

(46\%), IR $v_{\max } / \mathrm{cm}^{-1}: 1529\left(\mathrm{NO}_{2}\right), 1292\left(\mathrm{NO}_{2}\right), 1324$ (C-N); ${ }^{1} \mathrm{H}-\mathrm{NMR}\left(\mathrm{CDCl}_{3}\right) \delta 9.10(\mathrm{~d}, 1 \mathrm{H}, J 1.5 \mathrm{~Hz}, \mathrm{H}-2$ pyr.), 8.78 (d, 1H, J 4.8 Hz, H-4 pyr.), 8.23 (m, 1H, H-6 pyr.), 8.12 (d, 1H, J 6.5 Hz, H-3,5), 7.91 (d, 1H J 7.1 Hz, H-5-pyr.), 7.69-7.36 (m, 5H, Ar), 6.89 (d, 2H, J 9.5 Hz, H-2,6), 4.40 (t, $\left.2 \mathrm{H}, J 12.6 \mathrm{~Hz}, \mathrm{CH}_{2}-1\right), 3.15\left(\mathrm{t}, 4 \mathrm{H}, J 10.2 \mathrm{~Hz}, 2 \times \mathrm{CH}_{2}-\right.$ pip.), 2.85 (t, 2H, J $\left.12.6 \mathrm{~Hz}, \mathrm{CH}_{2}-2\right), 2.50$ (t, 4H, J 10.1 $\mathrm{Hz}, 2 \times \mathrm{CH}_{2}$ pip. $) .{ }^{13} \mathrm{C}-\mathrm{NMR}\left(\mathrm{CDCl}_{3}\right) \delta 154.6,150.7,149.9$, $147.5,137.2,134.2,130.8,2 \times 125.9,124.7,123.6,123.4$, $122.9,120.4,2 \times 112.8,110.1,58.4,57,32 \times 53.1,2 \times 47.9$, 39.8. Anal. Calc. for $\mathrm{C}_{24} \mathrm{H}_{24} \mathrm{~N}_{6} \mathrm{O}_{2}: \mathrm{C}, 67.27 ; \mathrm{H}, 5.65 ; \mathrm{N}$, 19.61. Found: C, 66.93; H, 5.42; N, $19.42 \%$.

1-\{2-[4-(3-Methoxyphenyl)piperazin-1-yl]ethyl\}-2pyridin-3-yl-1H-benzimidazole (5e)

(20\%), IR v max $/ \mathrm{cm}^{-1}: 2883(\mathrm{C}-\mathrm{H}), 1313(\mathrm{C}-\mathrm{N}) ;{ }^{1} \mathrm{H}-\mathrm{NMR}$ $\left(\mathrm{CDCl}_{3}\right) \delta 9.10$ (d, 1H, J $2.1 \mathrm{~Hz}, \mathrm{H}-2$ pyr.), 8.78 (d, 1H, J $4.9 \mathrm{~Hz}, \mathrm{H}-4$ pyr.), 8.22 (d, 1H, J 7.9 Hz, H-6 pyr.), 7.92 (d, $1 \mathrm{H} J$ 7.1Hz, H-5-pyr.), 7.62-7.12 (m, 5H, Ar), 6.61-6.21 (m, 3H, H-4,5,6), 4.40 (t, 2H, J6.6 Hz, $\left.\mathrm{CH}_{2}-1\right), 3.86(\mathrm{~s}, 3 \mathrm{H}$, $\mathrm{OCH}_{3}$ ), 3.15 (t, $4 \mathrm{H}, J 5.0 \mathrm{~Hz}, 2 \times \mathrm{CH}_{2}$-pip.), 2.84 (t, $2 \mathrm{H}, J 13$ $\mathrm{Hz}, \mathrm{CH}_{2}-2$ ), 2.58 (t, $4 \mathrm{H}, J 5.1 \mathrm{~Hz}, 2 \times \mathrm{CH}_{2}$ pip.). ${ }^{13} \mathrm{C}-\mathrm{NMR}$ $\left(\mathrm{CDCl}_{3}\right) \delta 160.6,152.4,150.7,149.9,147.2,143.2,137.2$, $135.6,129.8,127.3,123.6,123.4,122.9,120.3,110.2$, 108.9, 104.5, 102.6, 57.2, 55.2, 2×53.6, 2×48.9, 42.9. Anal. Calc. for $\mathrm{C}_{25} \mathrm{H}_{27} \mathrm{~N}_{5} \mathrm{O}: \mathrm{C}, 72.61 ; \mathrm{H}, 6.58 ; \mathrm{N}, 16.94$. Found: C, 72.76; H, 6.23; N, $16.82 \%$.

1-(2-(4-(Pyrazin-2-yl)piperazin-1-yl)ethyl)-2-(pyridin-3yl)-1H-benzo[d]imidazole (5f)

(38\%), IR $v_{\max } / \mathrm{cm}^{-1}: 2856(\mathrm{C}-\mathrm{H}), 1311(\mathrm{C}-\mathrm{N}) ;{ }^{1} \mathrm{H}-\mathrm{NMR}$ $\left(\mathrm{CDCl}_{3}\right) \delta 9.10$ (d, 1H, J $2.1 \mathrm{~Hz}, \mathrm{H}-2$ pyr.), 8.78 (d, 1H, J $4.8 \mathrm{~Hz}, \mathrm{H}-4$ pyr.), 8.36-8.21 (m, 2H, H-2 pyr., H-6 pyr.), 7.91 (d, 1H, J 9.0 Hz, H-5-pyr.), 7.76-7.31 (m, 5H, Ar), 6.50 (t, 1H, J 4.1 Hz, H-6), 4.42 (t, 2H, J 6.6 Hz, CH -1$)$, 3.17 (t, 4H, J $4.9 \mathrm{~Hz}, 2 \times \mathrm{CH}_{2}$-pip.), 2.83 (t, 2H, J $6.4 \mathrm{~Hz}$, $\left.\mathrm{CH}_{2}-2\right), 2.49$ (t, $4 \mathrm{H}, J 5 \mathrm{~Hz}, 2 \times \mathrm{CH}_{2}$ pip.). ${ }^{13} \mathrm{C}-\mathrm{NMR}\left(\mathrm{CDCl}_{3}\right)$ $\delta 162.3,157.7,156.2,150.7,149.9,147.5,143.3,137.1$, $135.6,129.8,127.5,123.4,122.9,120.3,110.7,110.0$, 57.3, $2 \times 53.4,2 \times 48.4,42.8$. Anal. Calc. for $\mathrm{C}_{22} \mathrm{H}_{23} \mathrm{~N}_{7}$ : C, 68.55; H, 6.01; N, 25.44. Found: C, 68.36; H, 6.26; $\mathrm{N}, 25.23 \%$.

1-[3-(4-Phenylpiperazin-1-yl)propyl]-2-pyridin-3-yl-1Hbenzimidazole $(5 \mathrm{~g})$

(48\%), IR $v_{\max } / \mathrm{cm}^{-1}: 2875(\mathrm{C}-\mathrm{H}), 1306(\mathrm{C}-\mathrm{N}) ;{ }^{1} \mathrm{H}-\mathrm{NMR}$ $\left(\mathrm{CDCl}_{3}\right) \delta 9.10$ (d, 1H, J $2.2 \mathrm{~Hz}, \mathrm{H}-2$ pyr.), 8.78 (d, 1H, $J 4.9 \mathrm{~Hz}, \mathrm{H}-4$ pyr.), 8.22 (d, 1H, J 8 Hz, H-6 pyr.), 7.89 (d, 1H, J 9.2 Hz, H-5-pyr.), 7.53-7.20 (m, 6H, Ar), 6.95 
(m, 3H, H-2,4,6), 4.40 (t, 2H, J $\left.7.8 \mathrm{~Hz}, \mathrm{CH}_{2}-1\right), 3.16$ (t, $4 \mathrm{H}, J 9.8 \mathrm{~Hz}, 2 \times \mathrm{CH}_{2}$-pip.), 2.50 (t, $4 \mathrm{H}, J 9.9 \mathrm{~Hz}, 2 \times \mathrm{CH}_{2}$ pip.), 2.37 (t, $\left.2 \mathrm{H}, J 6.6 \mathrm{~Hz}, \mathrm{CH}_{2}-3\right), 1.91\left(\mathrm{~m}, 2 \mathrm{H}, \mathrm{CH}_{2}-2\right)$. ${ }^{13} \mathrm{C}-\mathrm{NMR}\left(\mathrm{CDCl}_{3}\right) \delta 151.1,150.7,150.4,149.7,147.6$, 143.1, 136.9, 135.7, 134.4, 2×129.4, 127.6, 123.6, 123.1, 122.9, 120.1, 119.8, $2 \times 116.1,54.6,2 \times 53.1,2 \times 49,7,29.8$. Anal. Calc. for $\mathrm{C}_{25} \mathrm{H}_{27} \mathrm{~N}_{5}: \mathrm{C}, 75.54 ; \mathrm{H}, 6.85 ; \mathrm{N}, 17.62$. Found: C, 75.31; H, 6.90; N, 17.79\%.

1-\{3-[4-(2-Fluorophenyl)piperazin-1-yl]propyl\}-2-pyridin3-yl-1H-benzimidazole (5h)

(28\%), IR $v_{\max } / \mathrm{cm}^{-1}: 1225$ (C-N), 1007 (C-F); ${ }^{1} \mathrm{H}-\mathrm{NMR}\left(\mathrm{CDCl}_{3}\right) \delta 9.10$ (d, $1 \mathrm{H}, J 3 \mathrm{~Hz}, \mathrm{H}-2$ pyr.), 8.80 (d, $1 \mathrm{H}, J 6.6 \mathrm{~Hz}, \mathrm{H}-4$ pyr.), 8.23 (d, 1H, J 7.9 Hz, H-6 pyr.), 7.91 (d, 1H, J 5.3 Hz, H-5-pyr.), 7.61-7.21 (m, 6H, Ar), 7.12-6.86 (m, 2H, Ar), 4.41 (t, 2H, J 7.1 Hz, $\left.\mathrm{H} \mathrm{CH}_{2}-1\right), 3.20$ (t, $4 \mathrm{H}, J 4.5 \mathrm{~Hz}, \mathrm{CH}_{2}-2 \times \mathrm{CH}_{2}$-pip.), 2.50 (t, $4 \mathrm{H}, J 4.9 \mathrm{~Hz}$, $2 \times \mathrm{CH}_{2}$ pip.), 2.36 (t, $\left.2 \mathrm{H}, J 6.7 \mathrm{~Hz}, \mathrm{CH}_{2}-3\right), 1.97$ (t, $2 \mathrm{H}, J$ $\left.13.8 \mathrm{~Hz}, \mathrm{CH}_{2}-2\right) .{ }^{13} \mathrm{C}-\mathrm{NMR}\left(\mathrm{CDCl}_{3}\right) \delta 158.10\left(\mathrm{~d}, 2 \mathrm{C}, J_{\mathrm{C}-\mathrm{F}}\right.$ $318 \mathrm{~Hz}), 153.10,150.7,147.4,143.1,139.1,136.9,135.7$, $134.4,127.1,124.5,123.9,123.3,122.9,120.1,118.9$, $116.3,116.9,115.9,110.4,54.7,2 \times 53.2,2 \times 50,4,50.4$, 29.9. Anal. Calc. for $\mathrm{C}_{25} \mathrm{H}_{26} \mathrm{FN}_{5}: \mathrm{C}, 72.27 ; \mathrm{H}, 6.31 ; \mathrm{N}, 16.85$. Found: C, 71.99; H, 6.67; N, 16.49\%.

1-\{3-[4-(4-Chlorophenyl)piperazin-1-yl]propyl\}-2-pyridin3-yl-1H-benzimidazole (5i)

(38\%), IR $v_{\max } / \mathrm{cm}^{-1}: 1227(\mathrm{C}-\mathrm{N}), 933(\mathrm{C}-\mathrm{Cl}) ;{ }^{1} \mathrm{H}-\mathrm{NMR}$ $\left(\mathrm{CDCl}_{3}\right) \delta 9.10(\mathrm{~d}, 1 \mathrm{H}, J 2.5 \mathrm{~Hz}, \mathrm{H}-2$ pyr. $), 8.78(\mathrm{~d}, 1 \mathrm{H}$, $J 4.8 \mathrm{~Hz}, \mathrm{H}-4$ pyr.), 8.22 (d,1H, J $8.0 \mathrm{~Hz}, \mathrm{H}-6$ pyr.), 7.90 (d, $1 \mathrm{H} \mathrm{J} 7.4 \mathrm{~Hz}, \mathrm{H}-5$-pyr.), 7.52-7.14 (m, 6H, Ar), 7.116.87 (m, 2H, H-2,6), 4.41 (m, 2H, $\left.\mathrm{H} \mathrm{CH}_{2}-1\right), 3.15$ (m, 4H, $\mathrm{CH}_{2}-2 \times \mathrm{CH}_{2}$-pip.), 2.48 (m, $4 \mathrm{H}, 2 \times \mathrm{CH}_{2}$ pip.), 2.32 (m, $2 \mathrm{H}$, $\left.\mathrm{CH}_{2}-3\right), 2.0\left(\mathrm{~m}, 2 \mathrm{H}, \mathrm{CH}_{2}-2\right) .{ }^{13} \mathrm{C}-\mathrm{NMR}\left(\mathrm{CDCl}_{3}\right) \delta$ 151.6, $150.8,147.3,137.1,134.5,132.7,2 \times 129.0,127.5,124,2$ 123.6, 123.5, 123.3, 122.9, 120.3, 2x118.3, 110.2, 58.4, 57.1, 2×53.7, 2×49.1, 29.7. Anal. Calc. for: $\mathrm{C}_{25} \mathrm{H}_{26} \mathrm{ClN}_{5}$ : C, 69.51; H, 6.07; N, 16.21. Found: C, 69.27; H, 6.14; $\mathrm{N}, 15.99 \%$.

1-\{3-[4-(4-Nitrophenyl)piperazin-1-yl]propyl\}-2-pyridin3-yl-1H-benzimidazole (5j)

(24\%), IR V $v_{\max } / \mathrm{cm}^{-1}: 1530\left(\mathrm{NO}_{2}\right), 1296\left(\mathrm{NO}_{2}\right) .{ }^{1} \mathrm{H}-\mathrm{NMR}$ $\left(\mathrm{CDCl}_{3}\right) \delta 9.10(\mathrm{~d}, 1 \mathrm{H}, J 1.5 \mathrm{~Hz}, \mathrm{~d}, \mathrm{H}-2 \mathrm{pyr}$ ), $8.78(\mathrm{~d}, 1 \mathrm{H}$, $J 4.9 \mathrm{~Hz}, \mathrm{H}-4$ pyr.), 8.22 (m, 1H, H-6 pyr.), 8.13 (d, 1H, $J$ $6.7 \mathrm{~Hz}, \mathrm{H}-3,5), 7.90$ (d, 1H, J 6.9 Hz, H-5-pyr.), 7.68-7.36 (m, 5H, Ar), 6.89 (d, 2H, J 9.4 Hz, H-2,6), 4.40 (t, 2H, $J$ $7.1 \mathrm{~Hz}, \mathrm{CH}_{2}-1$ ), 3.23 (t, $4 \mathrm{H}, J 5.0 \mathrm{~Hz}, 2 \times \mathrm{CH}_{2}$-pip.), 2.48 (t, $4 \mathrm{H}, J 5.2 \mathrm{~Hz}, 2 \times \mathrm{CH}_{2}$ pip.), 2.35 (t, $2 \mathrm{H}, J 6.3 \mathrm{~Hz}, \mathrm{CH}_{2}-3$ ), 2.10 (bs, $\left.2 \mathrm{H}, J 6.8 \mathrm{~Hz}, \mathrm{CH}_{2}-2\right) .{ }^{13} \mathrm{C}-\mathrm{NMR}\left(\mathrm{CDCl}_{3}\right) \delta 154.6$, $150.7,149.9,147.5,137.2,136.7,134.2,130.8,2 \times 125.9$,
124.7, 123.6, 123.4, 122.9, 120.4, 2×112.8, 110.1, 58.4, $57,32 \times 53.1,2 \times 47.9,22.8$. Anal. Calc. for $\mathrm{C}_{25} \mathrm{H}_{26} \mathrm{~N}_{6} \mathrm{O}_{2}$ : C, 67.86; H, 5.92; N, 18.99. Found: C, 68.14; H, 5.69; N, $19.23 \%$.

1-\{3-[4-(3-Methoxyphenyl)piperazin-1-yl]propyl\}-2pyridin-3-yl-1H-benzimidazole (5k)

(33\%), IR $v_{\text {max }} / \mathrm{cm}^{-1}: 2835(\mathrm{C}-\mathrm{H}), 1315(\mathrm{C}-\mathrm{N}) ;{ }^{1} \mathrm{H}-\mathrm{NMR}$ $\left(\mathrm{CDCl}_{3}\right) \delta 9.10$ (d, $1 \mathrm{H}, J 2.19 \mathrm{~Hz}, \mathrm{H}-2$ pyr.), 8.78 (d, 1H, $J$ $4.9 \mathrm{~Hz}, \mathrm{H}-4$ pyr.), 8.21 (d, 1H, J 7.9 Hz, H-6 pyr.), 7.91 (d, $1 \mathrm{H}, J 5.5 \mathrm{~Hz}, \mathrm{H}-5$-pyr.), 7.63-7.13 (m, 5H, Ar), 6.61-6.24 (m, 3H, H-4,5,6), 4.40 (t, 2H, J 7.3 Hz, $\left.\mathrm{H} \mathrm{CH}_{2}-1\right), 3.86$ (s, $\left.3 \mathrm{H}, \mathrm{OCH}_{3}\right), 3.17$ (t, $4 \mathrm{H}, J 5.0 \mathrm{~Hz}, 2 \times \mathrm{CH}_{2}$-pip.), 2.50 (t, $4 \mathrm{H}$, $J 5 \mathrm{~Hz}, 2 \times \mathrm{CH}_{2}$ pip.), 2.36 (t, $2 \mathrm{H}, J 6.5 \mathrm{~Hz}, \mathrm{CH}_{2}-3$ ), 2.0 (b.s., $\left.2 \mathrm{H}, J 13.8 \mathrm{~Hz}, \mathrm{CH}_{2}-2\right) .{ }^{13} \mathrm{C}-\mathrm{NMR}\left(\mathrm{CDCl}_{3}\right) \delta 160.8,152.3$, 150.7, 150.0, 147.2, 143.3, 137.1, 136.6, 135.6, 129.8, 127.3, 123.6, 123.3, 122.9, 120.3, 110.4, 109.0, 104.6, 102.6, 57.3, 55.2, $2 \times 53.6,2 \times 48.9,22.9$. Anal. Calc. for $\mathrm{C}_{26} \mathrm{H}_{29} \mathrm{~N}_{5} \mathrm{O}: \mathrm{C}, 73.04 ; \mathrm{H}, 6.84 ; \mathrm{N}, 16.38$. Found: C, 72.89; $\mathrm{H}, 6.98 ; \mathrm{N}, 16.78 \%$.

\section{Acknowledgments}

This work was supported by VRAID (grant No. 01/2008).

\section{Supplementary Information}

Supplementary data are available free of charge at http:// jbcs.sbq.org.br, as PDF file.

\section{References}

1. Basavapathruni, A.; Anderson, K. S.; Curr. Pharm. Des. 2006, 12, 1857.

2. De Clercq, E.; Expert Opin. Emerg. Drug. 2005, 10, 241.

3. Persaud, D.; Gallant, J. E.; Hopkins HIV Rep. 2004, 16, 5.

4. Romero, D. L.; Morge, R. A.; Biles, C.; Berrios-Pena, N.; May, P. D.; Palmer, J. R.; Johnson, P. D.; Smith, H. W.; Busso, M.; Cheng-Keat, T.; Voorman, R. L.; Reusser, F.; Althaus, I. W.; Downey, K. M.; So, A. G.; Resnick, L.; Tarpley, W. G.; Aristoff, P. A.; J. Med. Chem. 1994, 37, 999.

5. Hajos, G.; Riedl, Z.; Molnar, J.; Szabo, D.; Drugs of the Future 2000, 25, 47.

6. Esnouf, R. M.; Ren, J.; Hopkins, A. L.; Ross, C. K.; Jones, E. Y.; Stammers, D. K.; Stuart, D. I.; Proc. Natl. Acad. Sci. U. S. A. 1997, 94, 3984.

7. Genin, M. J.; Poel, T. J.; Yagi, Y.; Biles, C.; Althaus, I.; Keiser, B. J.; Kopta, L. A.; Friis, J. M.; Reusser, F.; Adams, W. J.; Olmsted, R. A.; Voorman, R. L.; Thomas, R. C.; Romero, D. L.; J. Med. Chem. 1996, 39, 5267. 
8. Pinna, G.; Loriga, G.; Murineddu, G.; Grella, G.; Mura, M.; Vargiu, L.; Murgioni, C.; La Colla, P.; Chem. Pharm. Bull. 2001, 40, 1406.

9. Valderrama, J. A.; Pessoa-Mahana, H.; Sarrás, G.; Tapia, R.; Heterocycles 2001, 51, 2193.

10. (Comp. 2a) Hudkins, R. L.; Mailman, R. B.; DeHaven-Hudkins, D. L.; J. Med. Chem.1996, 37, 1964; (Comp. 2b) Konkel, M.; Wetzel, J. M.; Noble, S.; Gluchowski, C.; Craig, D. A.; PCT Int. Appl. 97 2000, CODEN: PIXXD2 WO 2000004012 A1
20000127; (Comp. 2c) Pollard, C. B.; Wicker, T. H., Jr.; J. Am. Chem. Soc. 1954, 76, 1853; (Comp. 2h) Kanno, T.; Gaino, M.; Yamamura, M.; Ishida, R.; Shintomi, K.; Eur. Pat. Appl. 89 1981, CODEN: EPXXDW EP 34284 A2 19810826; (Comps. 2g, 2i, 2k) Felfoldi, K.; Molnar, A.; Apjok, J.; Czombos, J.; Notheisz, F.; Karpati, E.; Acta Physica et Chemica 1982, 28, 225.

Received: February 5, 2009 Web Release Date: October 16, 2009 


\section{Synthesis of a Novel Series of 4-Arylpiperazinyl Derivatives Linked to a 2-(Pyridin-3-yl)-1H-benzimidazole as New Delavirdine Analogues}

\section{David Pessoa-Mahana, ${ }^{*, a}$ Andrés Núñez, ${ }^{a}$ Christian Espinosa, ${ }^{a}$ Jaime Mella-Raipán ${ }^{a}$ and Hernán Pessoa-Mahana ${ }^{b}$}

${ }^{a}$ Departamento de Farmacia, Facultad de Química, Pontificia Universidad Católica de Chile, Casilla 306, Santiago 22, Chile

${ }^{b}$ Departamento de Química Orgánica y Físico-Química, Facultad de Ciencias Químicas y Farmacéuticas, Universidad de Chile, Casilla 233, Santiago 1, Chile

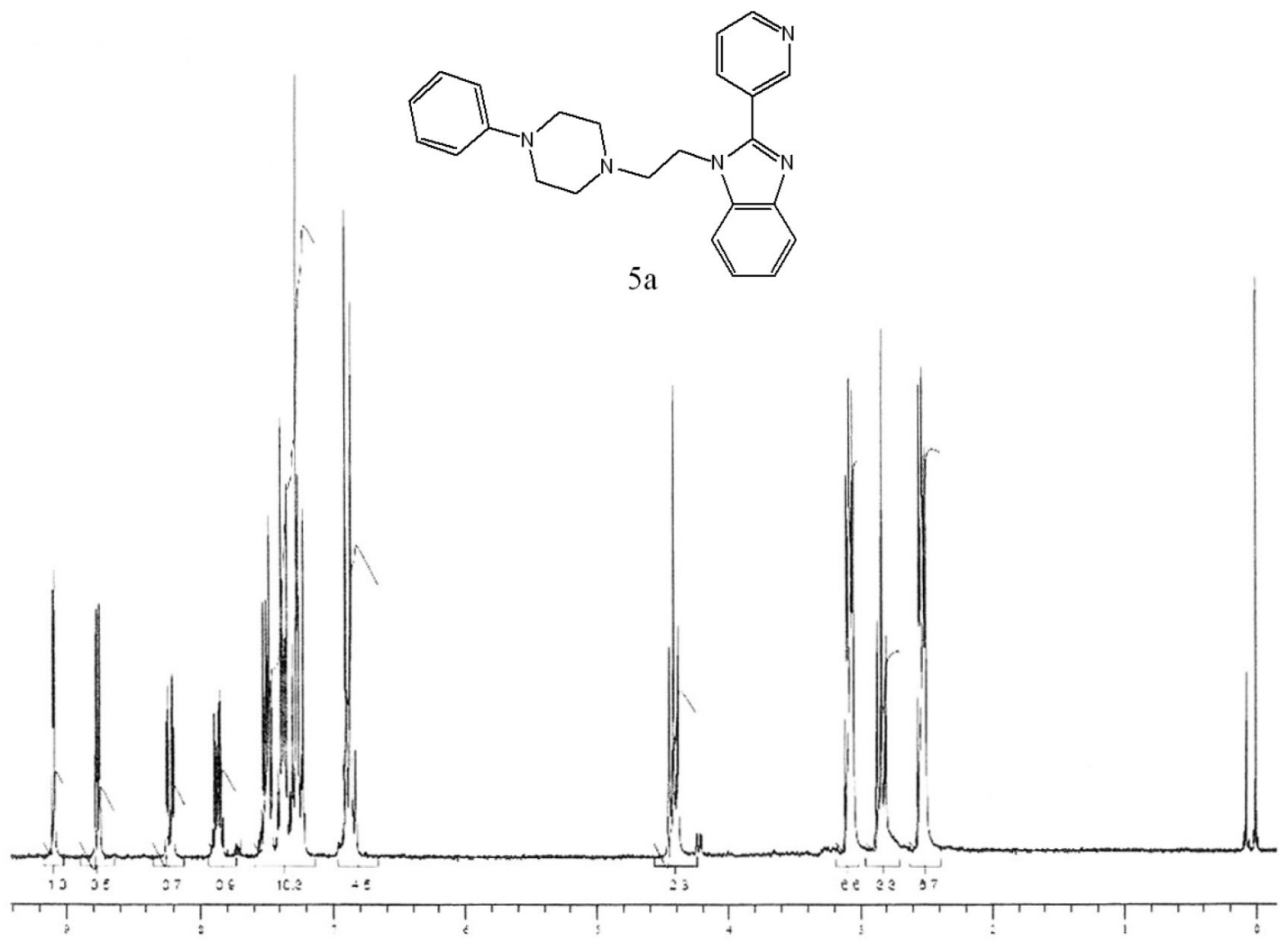

Figure S1. ${ }^{1} \mathrm{H}$ NMR spectrum of compound $\mathbf{5 a}\left(\mathrm{CDCl}_{3}, 200 \mathrm{MHz}\right)$. 


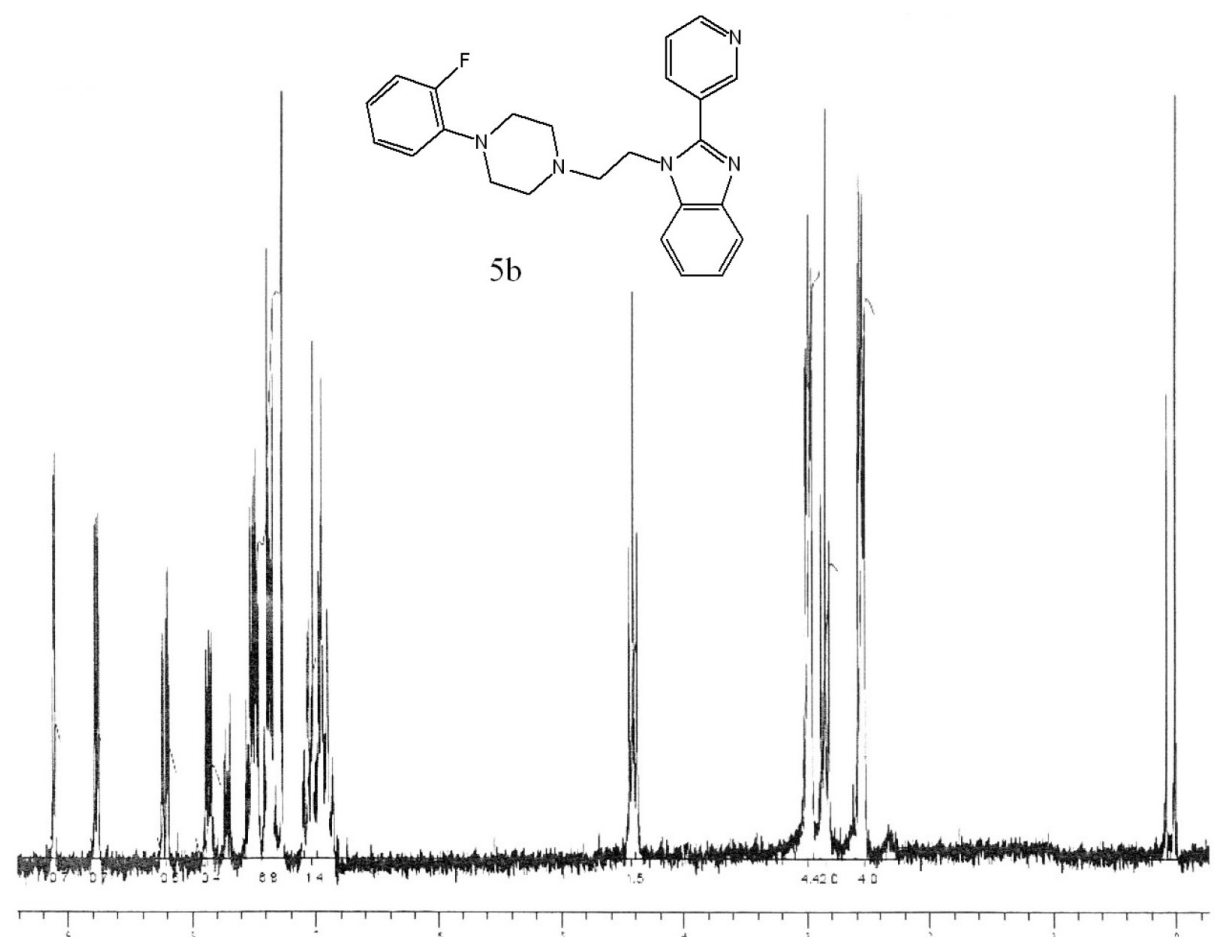

Figure S2. ${ }^{1} \mathrm{H}$ NMR spectrum of compound $\mathbf{5 b}\left(\mathrm{CDCl}_{3}, 200 \mathrm{MHz}\right)$.

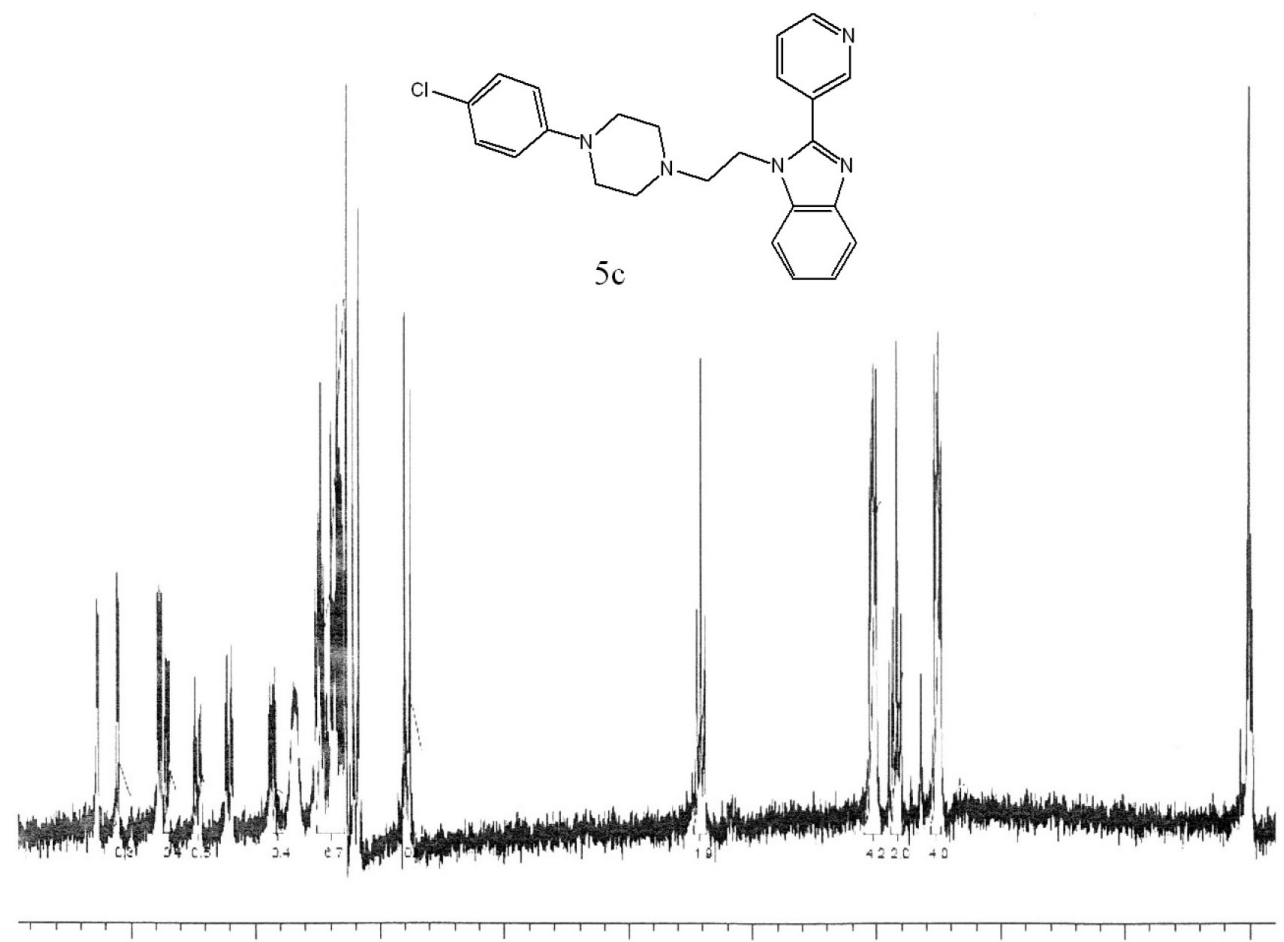

Figure S3. ${ }^{1} \mathrm{H}$ NMR spectrum of compound $\mathbf{5 c}\left(\mathrm{CDCl}_{3}, 200 \mathrm{MHz}\right)$. 


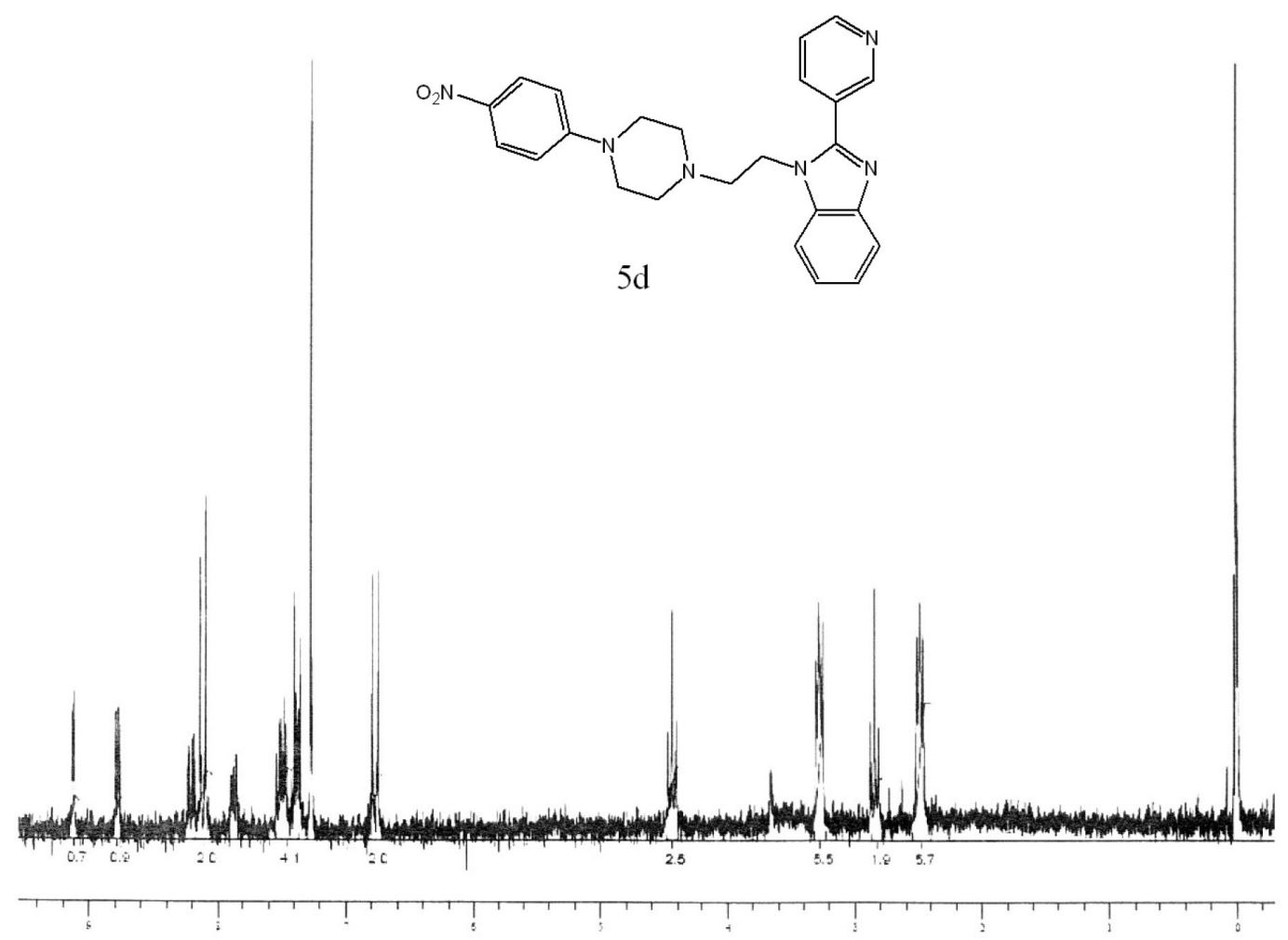

Figure S4. ${ }^{1} \mathrm{H}$ NMR spectrum of compound $\mathbf{5 d}\left(\mathrm{CDCl}_{3}, 200 \mathrm{MHz}\right)$.

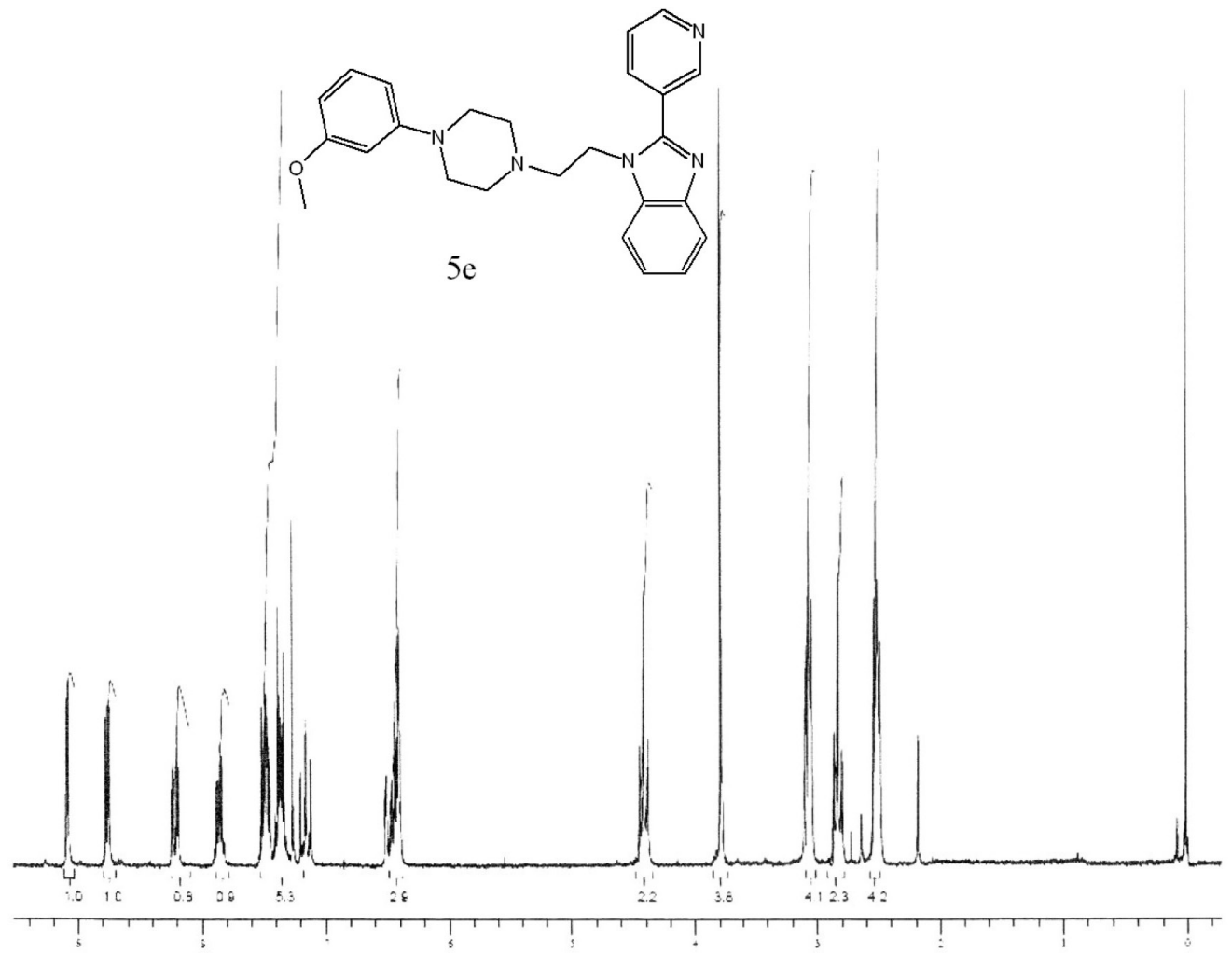

Figure S5. ${ }^{1} \mathrm{H}$ NMR spectrum of compound $\mathbf{5 e}\left(\mathrm{CDCl}_{3}, 200 \mathrm{MHz}\right)$. 


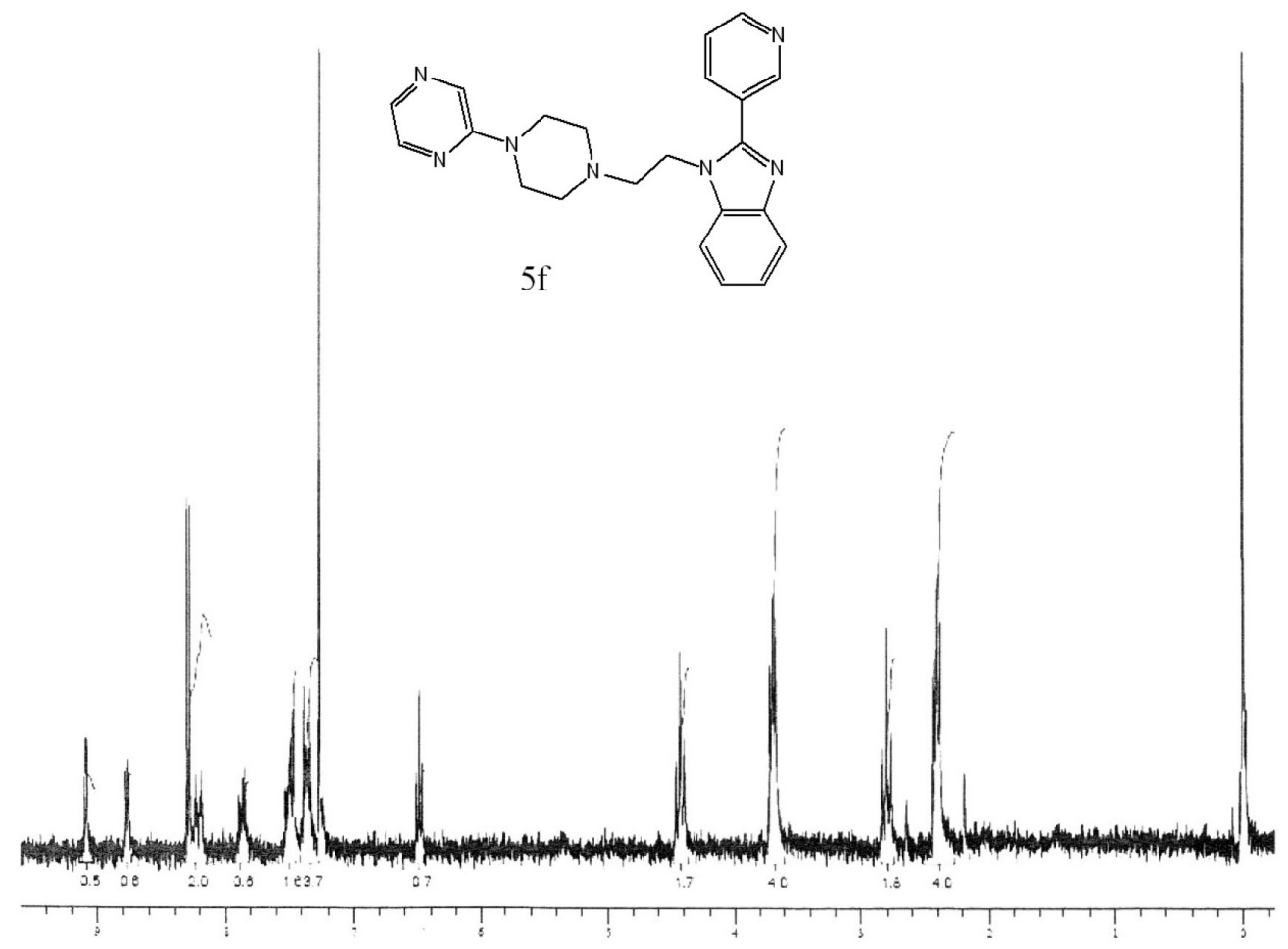

Figure S6. ' $\mathrm{H}$ NMR spectrum of compound $\mathbf{5 f}\left(\mathrm{CDCl}_{3}, 200 \mathrm{MHz}\right)$.

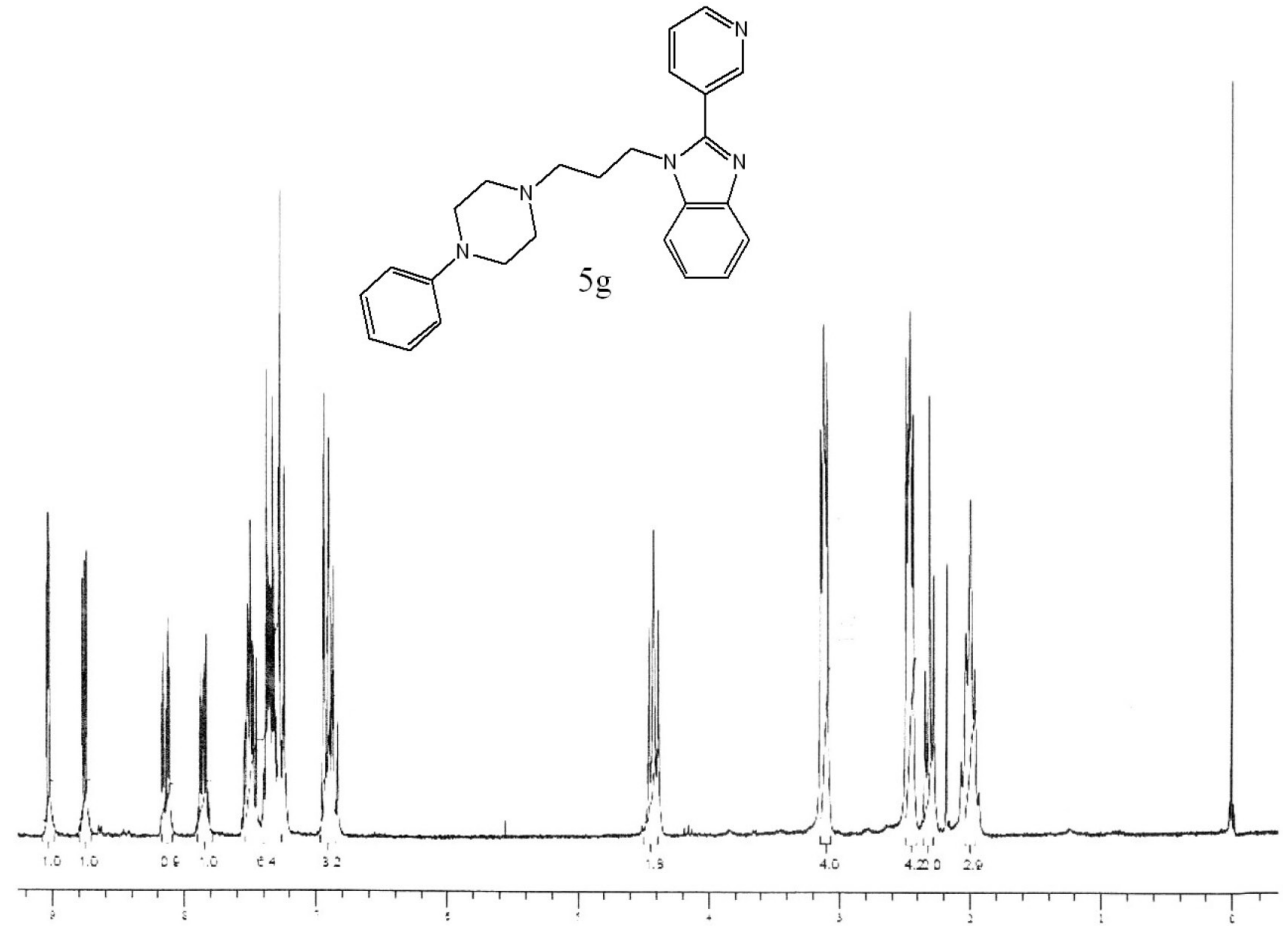

Figure S7. 'H NMR spectrum of compound $\mathbf{5 g}\left(\mathrm{CDCl}_{3}, 200 \mathbf{M H z}\right)$. 


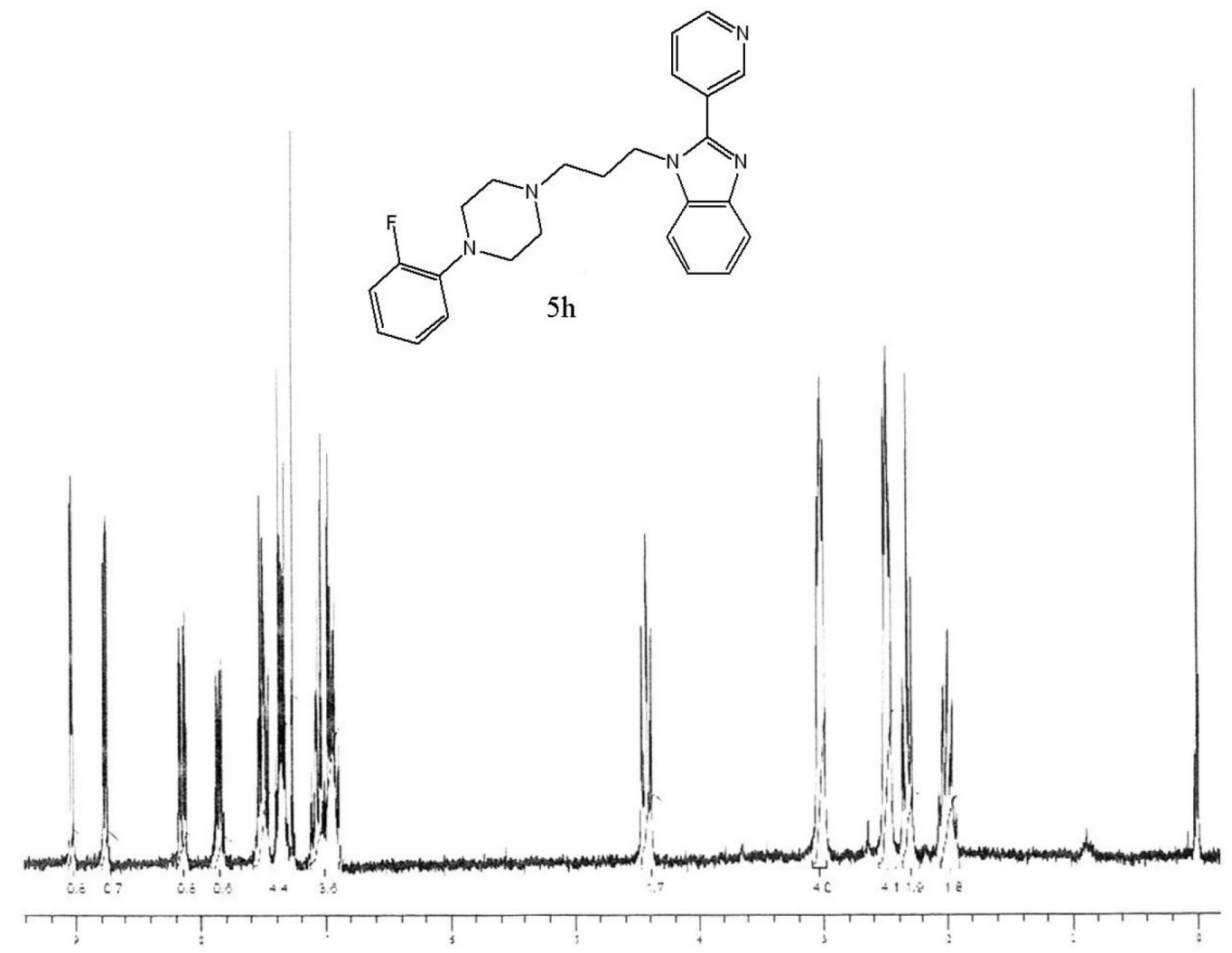

Figure S8. ${ }^{1} \mathrm{H}$ NMR spectrum of compound $\mathbf{5 h}\left(\mathrm{CDCl}_{3}, 200 \mathrm{MHz}\right)$.

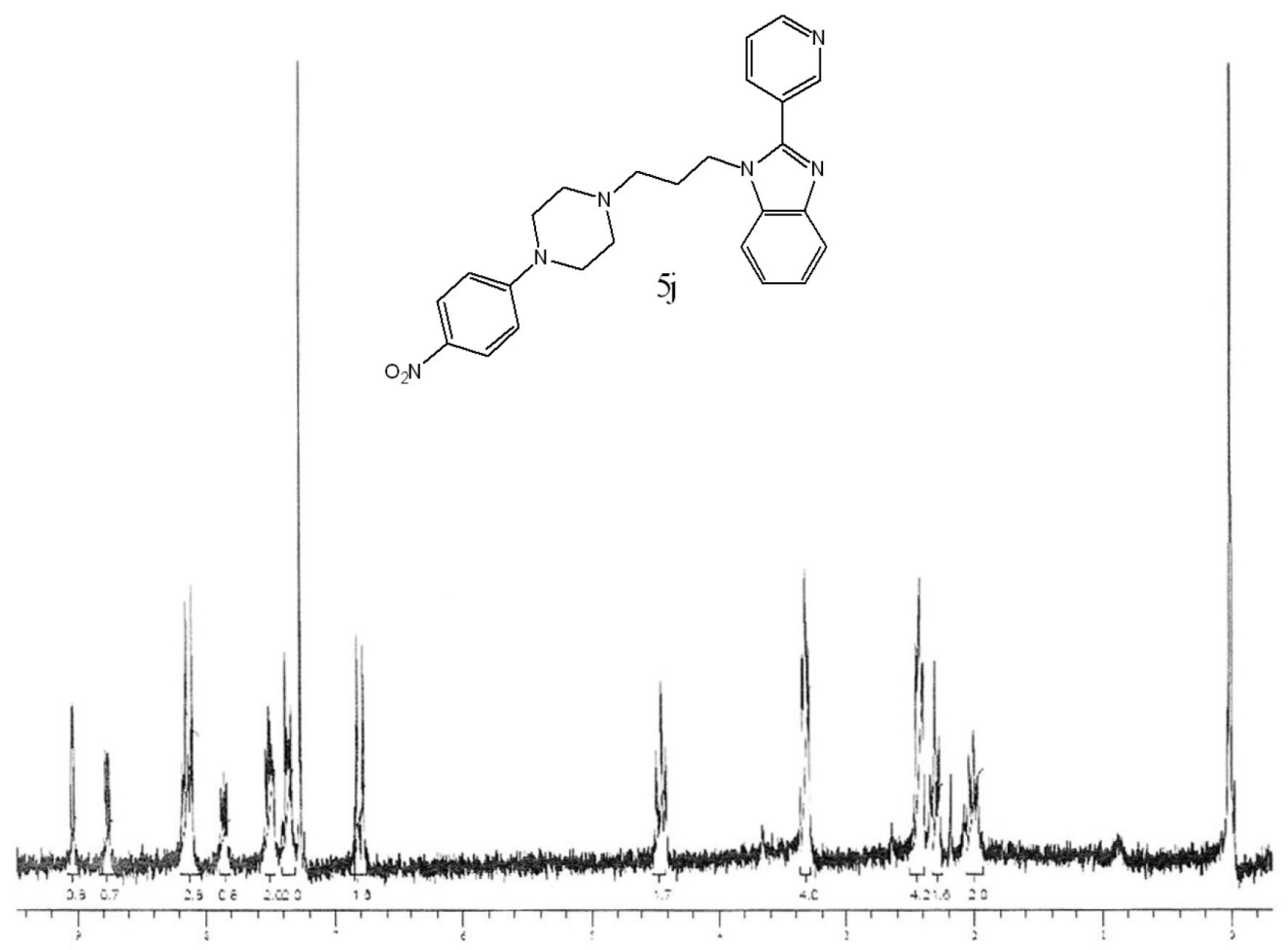

Figure S9. ' $\mathrm{H}$ NMR spectrum of compound $\mathbf{5 j}\left(\mathrm{CDCl}_{3}, 200 \mathrm{MHz}\right)$. 


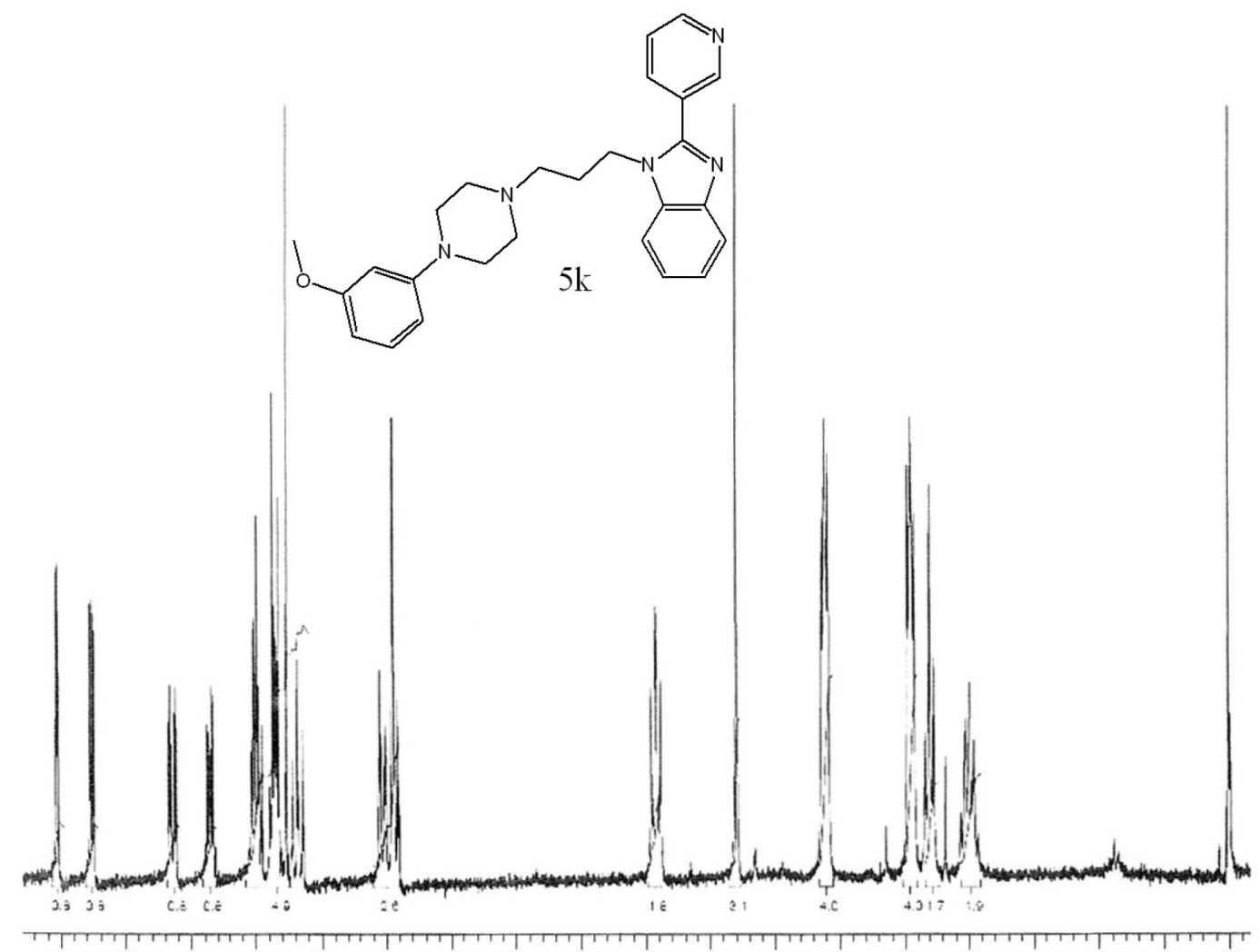

Figure S10. ${ }^{1} \mathrm{H}$ NMR spectrum of compound $\mathbf{5 k}\left(\mathrm{CDCl}_{3}, 200 \mathrm{MHz}\right)$.
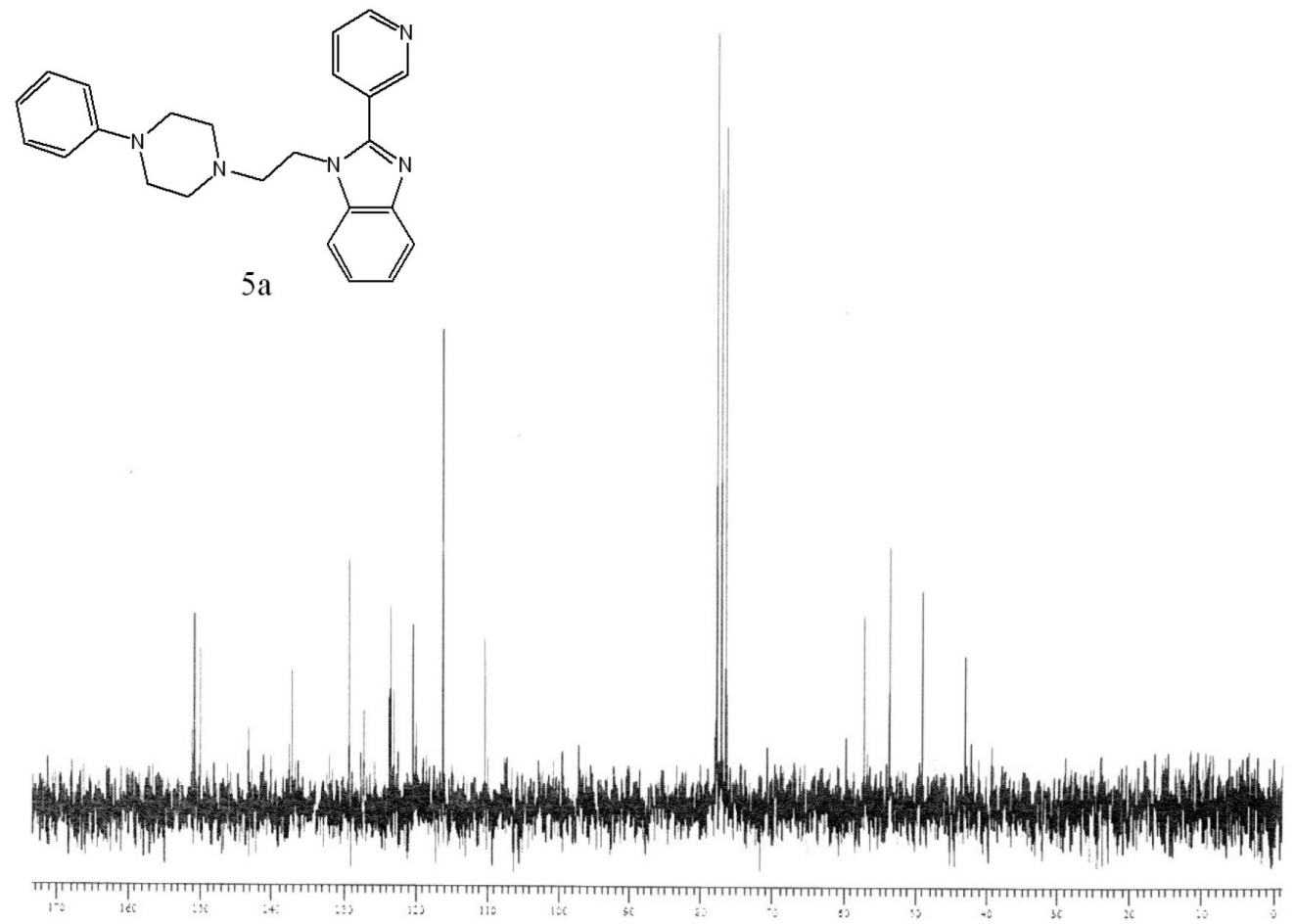

Figure S11. ${ }^{13} \mathrm{C}$ NMR spectrum of compound $\mathbf{5 a}\left(\mathrm{CDCl}_{3}, 50 \mathrm{MHz}\right)$. 
<smiles>COc1cccc(N2CCN(CCn3c(-c4cccnc4)nc4ccccc43)CC2)c1</smiles>
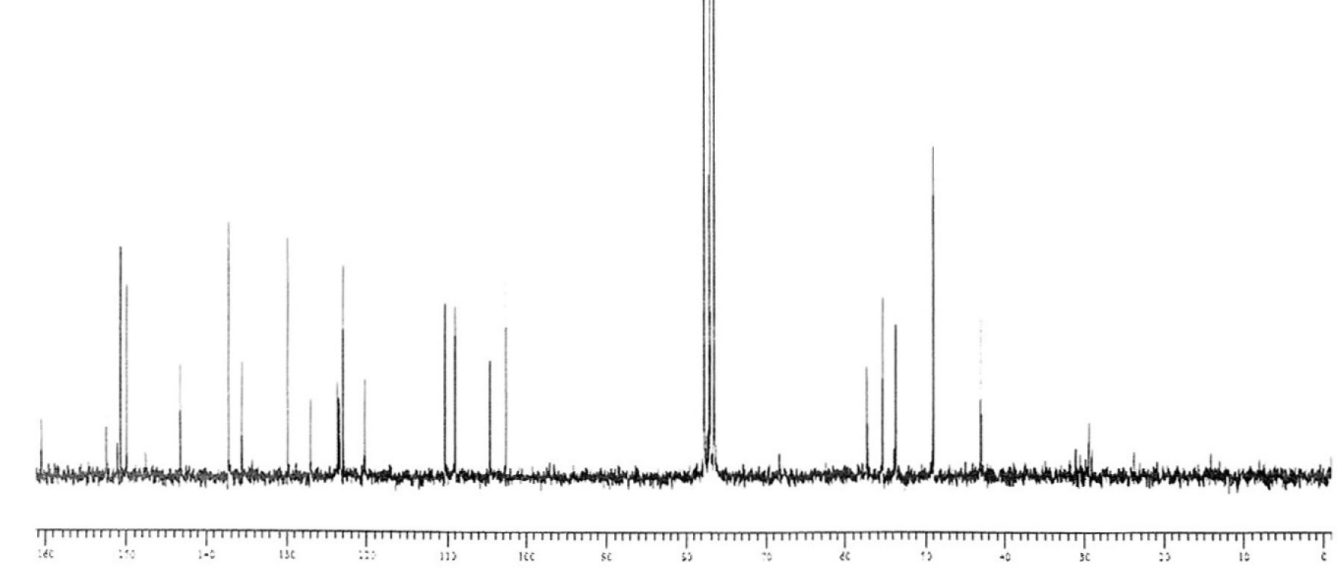

Figure S12. ${ }^{13} \mathrm{C}$ NMR spectrum of compound $\mathbf{5 e}\left(\mathrm{CDCl}_{3}, 50 \mathrm{MHz}\right)$.<smiles>c1cncc(-c2nc3ccccc3n2CCN2CCN(c3cnccn3)CC2)c1</smiles>

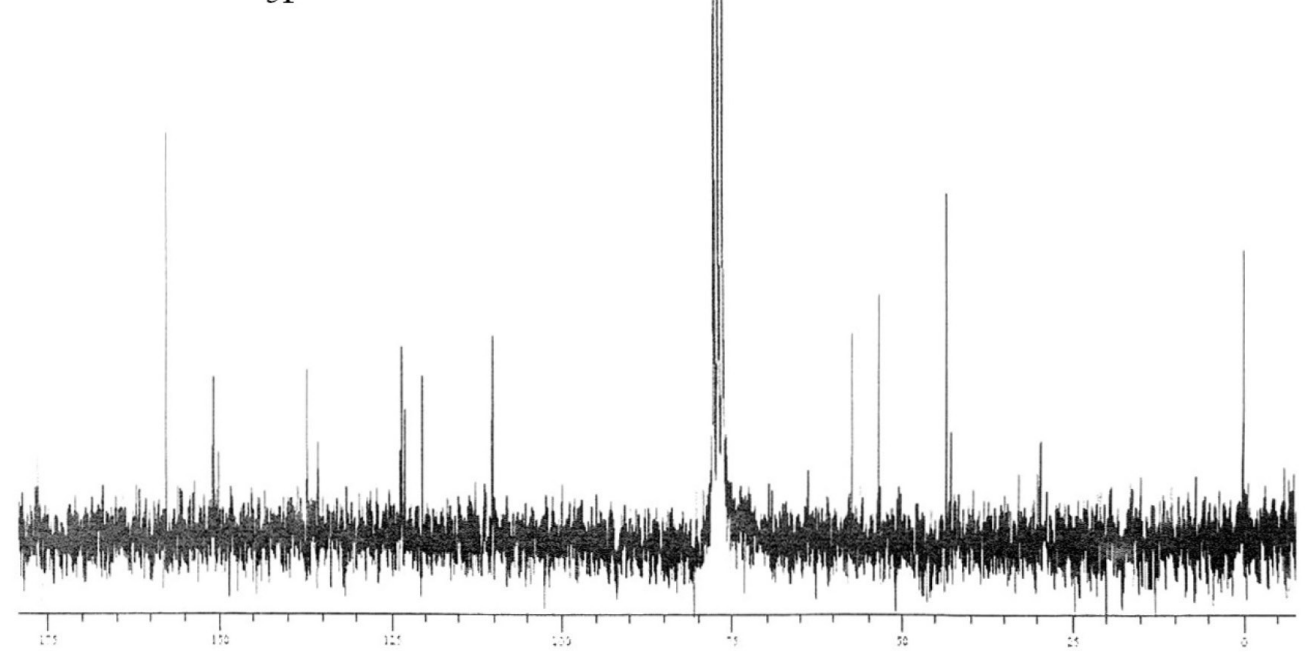

Figure S13. ${ }^{13} \mathrm{C}$ NMR spectrum of compound $\mathbf{5 f}\left(\mathrm{CDCl}_{3}, 50 \mathrm{MHz}\right)$. 

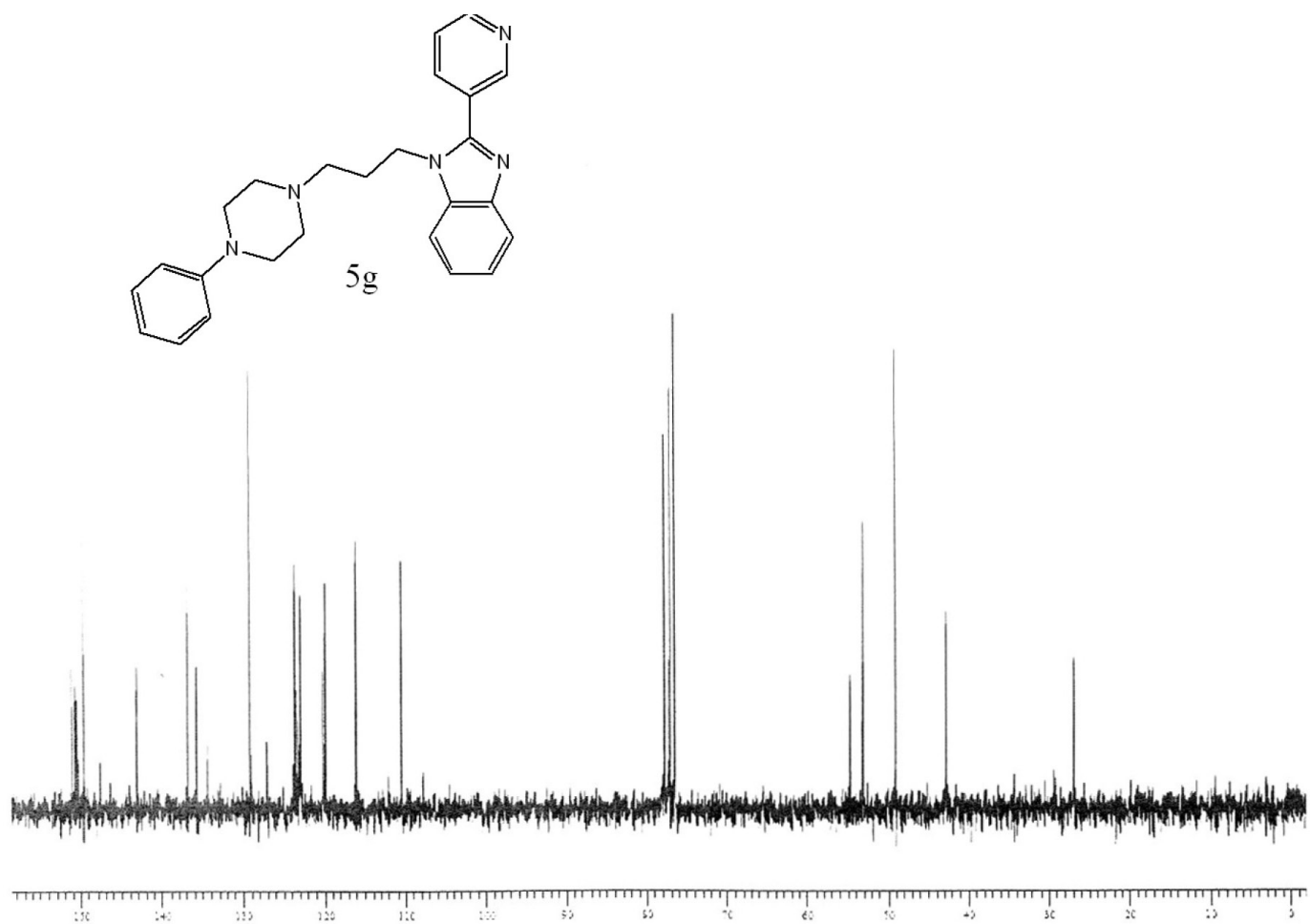

Figure S14. ${ }^{13} \mathrm{C}$ NMR spectrum of compound $5 \mathrm{~g}\left(\mathrm{CDCl}_{3}, 50 \mathrm{MHz}\right)$.
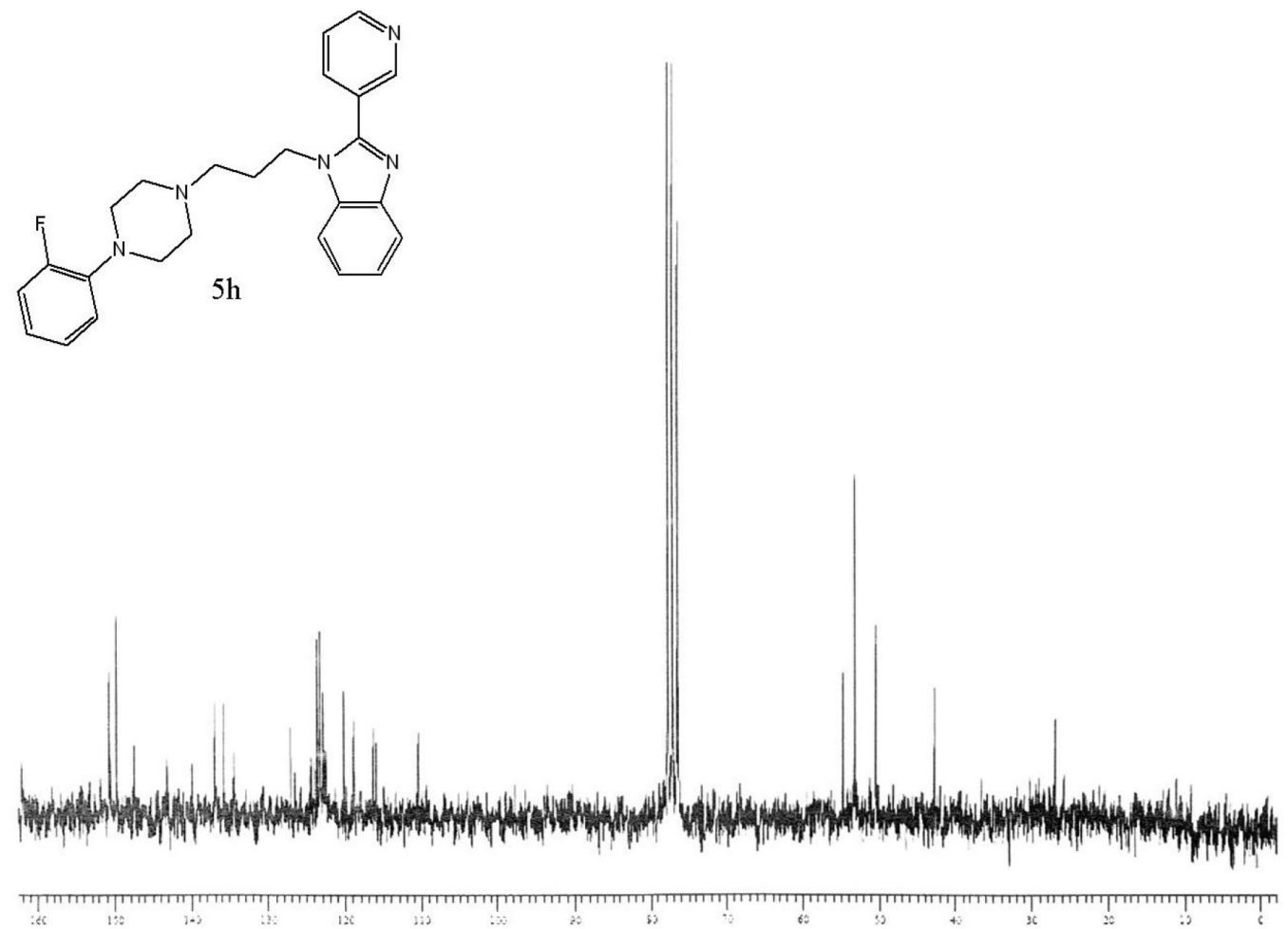

Figure S15. ${ }^{13} \mathrm{C}$ NMR spectrum of compound $\mathbf{5 h}\left(\mathrm{CDCl}_{3}, 50 \mathrm{MHz}\right)$. 


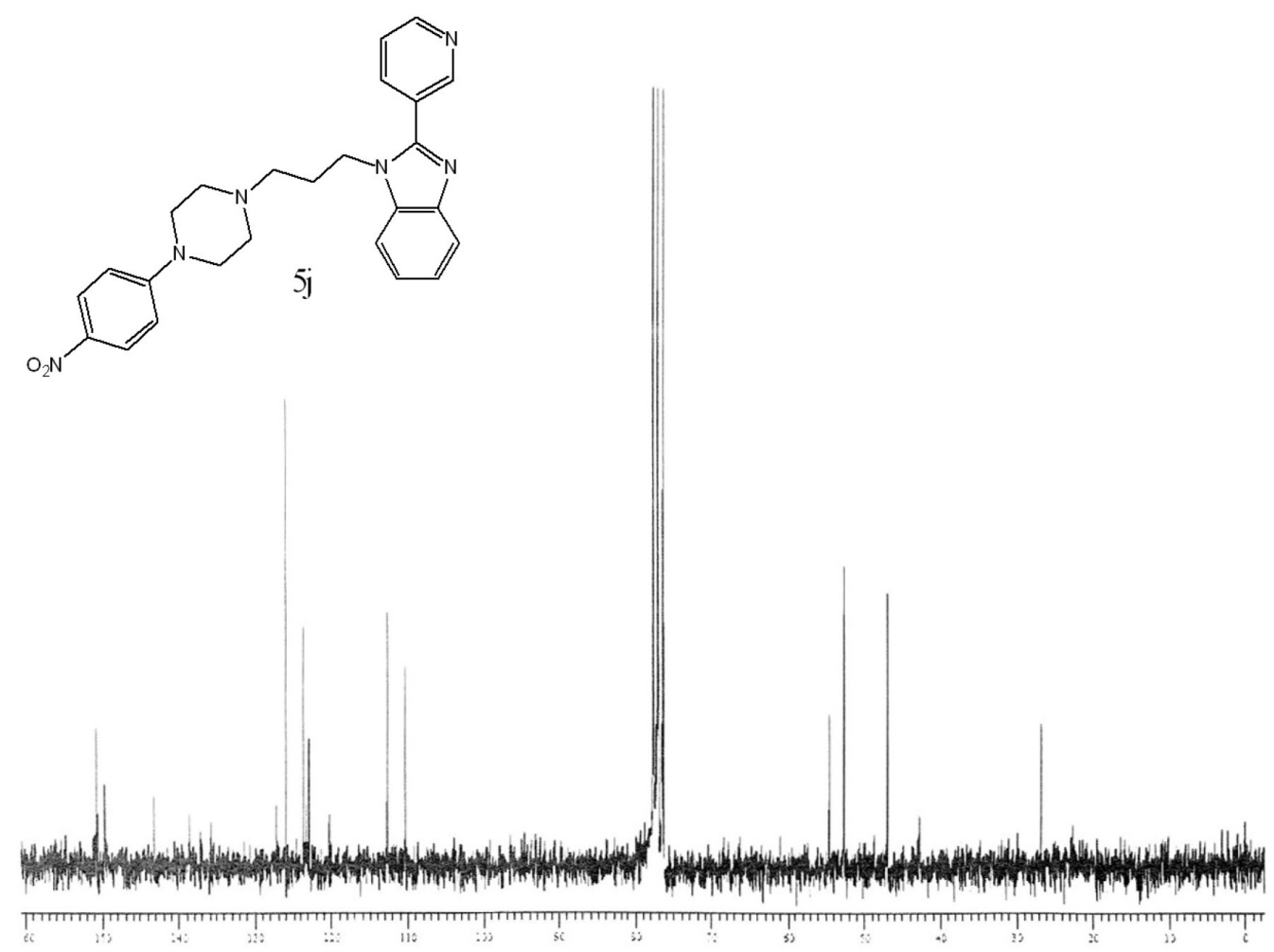

Figure S16. ${ }^{13} \mathrm{C}$ NMR spectrum of compound $\mathbf{5 j}\left(\mathrm{CDCl}_{3}, 50 \mathrm{MHz}\right)$. 